\title{
A CALCULUS OF LAX FRACTIONS
}

\author{
LURDES SOUSA
}

\begin{abstract}
Aвstract. We present a notion of category of lax fractions, where lax fraction stands for a formal composition $s_{*} f$ with $s_{*} s=$ id and $s s_{*} \leq$ id, and a corresponding calculus of lax fractions which generalizes the Gabriel-Zisman calculus of fractions.
\end{abstract}

\section{Introduction}

Given a class $\sum$ of morphisms of a category $X$, we can construct a category of fractions $X\left[\Sigma^{-1}\right]$ where all morphisms of $\Sigma$ are invertible. More precisely, we can define a functor $P_{\Sigma}: X \rightarrow X\left[\Sigma^{-1}\right]$ which takes the morphisms of $\Sigma$ to isomorphisms, and, moreover, $P_{\Sigma}$ is universal with respect to this property. As shown in [13], if $\sum$ admits a calculus of fractions, then the morphisms of $X\left[\Sigma^{-1}\right]$ can be expressed by equivalence classes of cospans $(f, g)$ of morphisms of $X$ with $g \in \Sigma$, which correspond to the formal compositions $g^{-1} f$.

We recall that categories of fractions are closely related to reflective subcategories and orthogonality. In particular, if $\mathcal{A}$ is a full reflective subcategory of $\mathcal{X}$, the class $\sum$ of all morphisms inverted by the corresponding reflector functor - equivalently, the class of all morphisms with respect to which $\mathcal{A}$ is orthogonal - admits a left calculus of fractions; and $\mathcal{A}$ is, up to equivalence of categories, a category of fractions of $X$ for $\Sigma$. In [3] we presented a Finitary Orthogonality Deduction System inspired by the left calculus of fractions, which can be looked as a generalization of the Implicational Logic of [20], see [4].

Assume now that $X$ is an order-enriched category, that is, its hom-sets $X(X, Y)$ are endowed with a partial order satisfying the condition $f \leq g \Rightarrow h f j \leq h g j$ for every morphisms $f, g: X \rightarrow Y$, $j: Z \rightarrow X$ and $h: Y \rightarrow W$. We call a morphism $f: X \rightarrow Y$ of $X$ a left adjoint section if it is a left adjoint and has a left inverse; equivalently, there is a morphism $f_{*}: Y \rightarrow X$ such that $f_{*} f=\operatorname{id}_{X}$ and $f f_{*} \leq \operatorname{id}_{Y}$. We are interested in a category of lax fractions in the sense that, given a class $\Sigma$ of morphisms of $X$, we want a category $X\left[\Sigma_{*}\right]$ and an order-enriched functor $P_{\Sigma}: X \rightarrow X\left[\Sigma_{*}\right]$ which takes morphisms of $\Sigma$ to left adjoint sections of $X\left[\Sigma_{*}\right]$ and, moreover, $P_{\Sigma}$ is universal with respect to that property. This problem is connected with the study of KZ-monads and Kan-injectivity as explained next.

In recent papers $([1,8])$ we have studied a lax version of orthogonality in order-enriched categories: Kan-injectivity. An object $A$ is said to be (left) Kan-injective with respect to a morphism $h: X \rightarrow Y$ provided that for every morphism $f: X \rightarrow A$ there is a left Kan extension of $f$ along $h$, denoted $f / h$, and, moreover, $f=(f / h) h$. And a morphism $k: A \rightarrow B$ is said to be Kan-injective with respect to $h$ if $A$ and $B$ are so and $k$ preserves left Kan extensions along $h$, i.e., $(k f) / h=k(f / h)$. Let $\mathcal{A}$ be a subcategory of an order-enriched category $X$. We say that $\mathcal{A}$ is KZ-reflective if it is

Date: June 6, 2016.

This work was partially supported by the Centre for Mathematics of the University of Coimbra UID/MAT/00324/2013, funded by the Portuguese Government through FCT/MEC and co-funded by the European Regional Development Fund through the Partnership Agreement PT2020. 
reflective and the monad induced in $X$ by the reflector functor $F: X \rightarrow \mathcal{A}$ is a KZ-monad, i.e., the unit $\eta$ satisfies the inequalities $F \eta_{X} \leq \eta_{F X}$ for all objects $X$ of $\mathcal{X}([18,12])$. If, moreover, $\mathcal{A}$ is an Eilenberg-Moore category of a KZ-monad over $X$, we say that $\mathcal{A}$ is a $\mathrm{KZ}$-monadic subcategory of $X$. Let $\mathcal{A}^{\text {Llnj }}$ denote the class of all morphisms with respect to which all objects and morphisms of $\mathcal{A}$ are Kan-injective. As shown in [8], if $\mathcal{A}$ is KZ-reflective in $X, \mathcal{A}^{\text {Llnj }}$ consists precisely of all morphisms of $\mathcal{X}$ whose images through the reflector functor are left adjoint sections.

In this paper we present the notion of category of lax fractions $P_{\Sigma}: X \rightarrow X\left[\Sigma_{*}\right]$ and a calculus of lax fractions which generalize the usual non-lax versions. But now $\sum$ is not just a class of morphisms, as in the ordinary case; instead, it is a subcategory of the arrow category $X \rightarrow$. And the calculus of lax fractions is expressed as a calculus of squares (called $\Sigma$-squares) which represent formal equalities of the form $f r_{*}=s_{*} g$ (see Section 4). This way, we obtain a description of the category of lax fractions of $X$, for $\sum$ a subcategory of $X \rightarrow$ admitting a left calculus of lax fractions,

in terms of formal fractions $s_{*} f$ represented by cospans $\stackrel{f}{\longrightarrow} \bullet<s$ (Theorem 4.11). The idea of "calculating" with squares of the base category $X$ instead of just with morphisms of $X$ is also used in the paper in preparation [2] in order to obtain a Kan-Injectivity Logic generalizing the Orthogonality Logic of [3].

Given a subcategory $\mathcal{A}$ of $X$, let $\mathcal{A} \underline{\text { Linj }}$ denote the subcategory of $X \rightarrow$ whose objects are the morphisms of $\mathcal{A}^{\text {Llnj }}$, and whose morphisms between them are those of the form $(u, v):(s: X \rightarrow Y) \longrightarrow$ $\left(s^{\prime}: Z \rightarrow W\right)$ such that $(f u) / s=\left(f / s^{\prime}\right) v$ for all $f$ with domain $Z$ and codomain in $\mathcal{A}$. We show that, for $\Sigma=\mathcal{A} \underline{\underline{L} \mathrm{nj}}$, if $\mathcal{A}$ is a KZ-reflective subcategory of $\mathcal{X}$, the category $\mathcal{X}\left[\Sigma_{*}\right]$ is the Kleisli category for the monad induced by the reflector functor $F: X \rightarrow \mathcal{A}$, and $F$ differs from the functor $P_{\Sigma}: X \rightarrow X\left[\Sigma_{*}\right]$ at most by closedness under left adjoint retractions (Theorem 3.7); moreover, $\Sigma$ admits a left calculus of lax fractions (Proposition 4.5).

We finish up with some properties on cocompleteness. We show that whenever $X$ has weighted colimits, any subcategory of $X^{\rightarrow}$ of the form $\Sigma=\mathcal{A}$ ㄴǌ also has weighted colimits (Theorem 5.1) and admits a left calculus of lax fractions, and the corresponding category of lax fractions $X\left[\Sigma_{*}\right]$ has (small) conical coproducts. Moreover, we present conditions on any subcategory $\Sigma$ under which $X\left[\Sigma_{*}\right]$ has finite conical coproducts, provided $X$ has them.

Several examples of subcategories $\Sigma$ of $X \rightarrow$ admitting a left calculus of lax fractions are provided in Example 4.4 for $X$ the category Pos of posets and monotone maps, the category Loc of locales and localic maps, and the category $\mathrm{Top}_{0}$ of $T_{0}$ topological spaces and continuous maps.

The study of constructions of categories by freely adding adjoints to the arrows of a category has been addressed before. Although the present approach is completely different, it is worth mentioning here the works [10] and [11] of Dawson, Paré and Pronk.

\section{Preliminaries}

Along this paper we work in the order-enriched context. More precisely, we consider categories and functors enriched in the category Pos of posets and monotone maps. For a category $X$ this means that each one of its hom-sets $X(X, Y)$ is equipped with a partial order $\leq$ which is preserved by composition on the left and on the right. And a functor between order-enriched categories is order-enriched if it preserves the partial order of the morphisms. A subcategory of an order-enriched category $X$ will be considered order-enriched via the restriction of the order on the morphisms of $X$ to the morphisms of $\mathcal{A}$. 
In this section, we recall the notions of Kan-injectivity and KZ-reflective subcategory, and some of their properties, which are presented in [8] and [1].

2.1. Kan-injectivity. In an order-enriched category $X$, an object $A$ is said to be left Kan-injective (or just Kan-injective) with respect to a morphism $h: X \rightarrow Y$, if, for every morphism $f: X \rightarrow A$, there is a morphism $f / h: Y \rightarrow A$ such that

(i) $(f / h) h=f$, and

(ii) $f \leq g h \Rightarrow f / h \leq g$, for every morphism $g: Y \rightarrow A$.

A morphism $k: A \rightarrow B$ is said to be (left) Kan-injective with respect to $h$ provided that $A$ and $B$ are so, and the equality $(k f) / h=k(f / h)$ holds for all $f: X \rightarrow A$.

(Left) Kan-injectivity may be equivalently defined as follows: An object $A$ is left Kan-injective with respect to a morphism $h: X \rightarrow Y$, if and only if the hom-map $X(h, A): X(Y, A) \rightarrow X(X, A)$ is a right adjoint retraction (short for a morphism which is simultaneously a right adjoint and a retraction) in the category Pos. In this case, if $(X(h, A))^{*}: X(X, A) \rightarrow X(Y, A)$ is the left adjoint of $X(h, A)$, then we have that $(X(h, A))^{*}(f)=f / h$.

Given a class $\mathcal{H}$ of morphisms of $\mathcal{X}$, the objects and morphisms of $X$ which are (left) Kaninjective with respect to all morphisms of $\mathcal{H}$ constitute a subcategory, denoted by

$$
\operatorname{LInj}(\mathcal{H})
$$

and said to be a Kan-injective subcategory ${ }^{1}$. And, given a subcategory $\mathcal{A}$ of $\mathcal{X}$, we denote by

$$
\mathcal{A}^{\text {LInj }}
$$

the class of all morphisms with respect to which all objects and morphisms of $\mathcal{A}$ are Kan-injective.

2.2. KZ-reflective subcategories. We recall that a KZ-monad (or lax idempotent monad) on $X$ is a monad $T: X \rightarrow X$ whose unit $\eta$ satisfies the inequalities $T \eta_{X} \leq \eta_{T X}, X \in X$ ([18], [12]). Let $\mathcal{A}$ be a subcategory of $X . \mathcal{A}$ is said to be a KZ-reflective subcategory of $X$ if it is reflective in $X$ and the monad over $X$ induced by the corresponding adjunction is of $\mathrm{KZ}$ type; that is, the left adjoint $F: X \rightarrow \mathcal{A}$ and the unit $\eta$ satisfy the inequalities

$$
F \eta_{X} \leq \eta_{F X}, X \in X .
$$

The Eilenberg-Moore categories of KZ-monads over $X$ are, up to isomorphism of categories, KZreflective subcategories, called then KZ-monadic subcategories. Thus the concept of KZ-monadic subcategory is a lax version of the one of replete full reflective subcategory. In [8] we showed that KZ-monadic subcategories are precisely the KZ-reflective categories closed under left adjoint retractions (i.e., the equality $g x=y f$ between morphisms of $X$ with $f$ in $\mathcal{A}$ and $x$ and $y$ both left adjoint retractions implies that $g$ also belongs to $\mathcal{A}$ ). In [1] we proved that in well-behaved categories, namely in locally ranked ones, every Kan-injective subcategory LInj( $\mathcal{H})$ with $\mathcal{H}$ a set is indeed a KZ-monadic subcategory.

When $\mathcal{A}$ is KZ-reflective in $X$, with $F: X \rightarrow \mathcal{A}$ the corresponding reflector functor, $\mathcal{A}^{\text {Llnj }}$ is precisely the class of all morphisms $f$ of $X$ such that $F f$ is a left adjoint section in $\mathcal{A}$, that is, there is a morphism $(F h)_{*}$ in $\mathcal{A}$ with $(F h)_{*} F h=\mathrm{id}$ and $F h(F h)_{*} \leq \mathrm{id}([8])$. We call this kind of morphisms F-embeddings, following the terminology of M. Escardó [12].

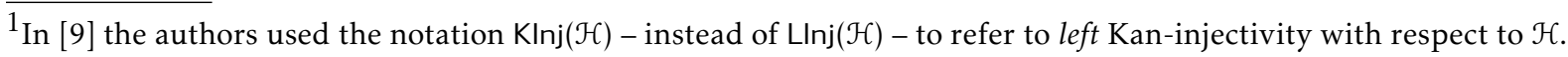




\section{Categories of lax fractions}

It is well known that if $\mathcal{A}$ is a full reflective subcategory of an ordinary category $X$ with reflector functor $F: X \rightarrow \mathcal{A}$, then $\mathcal{A}$ is, up to equivalence of categories, the category of fractions of $X$ for the class of morphisms inverted by $F$. Indeed, this category of fractions is the Kleisli category of the idempotent monad induced by the corresponding adjunction. Formally we can think of a "fraction" as a composition of the form $h^{-1} f$ where $h^{-1}$ is a formal inverse of $h$. Here we use the term "lax fraction" evoking a composition of the form $h_{*} f$ where $h_{*}$ is a formal left inverse and right adjoint of $h$ (that is, $h_{*}$ is thought as satisfying $h_{*} h=\mathrm{id}$ and $\mathrm{id} \leq h_{*} h$ ). We show that, in the order-enriched context, a KZ-reflective subcategory $\mathcal{A}$ of $\mathcal{X}$, with reflector $F: \mathcal{X} \rightarrow \mathcal{A}$, is also closely related to the category of lax fractions of $X$ for the $F$-embeddings of $X$. And this category of lax fractions coincides with the Kleisli category of the corresponding KZ-monad too.

Given a full subcategory $\mathcal{A}$ of any category $X$, some of the nice properties of the class $\mathcal{A}^{\text {Orth }}$ of all morphisms with respect to which $\mathcal{A}$ is orthogonal are obtained by looking at $\mathcal{A}^{\text {Orth }}$ as a full subcategory of the arrow category $X \rightarrow$. This is the case, for instance, of the closedness under colimits of $\mathcal{A}^{\text {Orth }}$ in $X \rightarrow$, when $X$ is cocomplete (cf. [21]). Let $X$ be an order-enriched category, and let $X \rightarrow$ be order-enriched with the coordinatewise order. KZ-reflective subcategories are not full, in general. Thus it is not surprising that, in order to generalize orthogonality properties to Kan-injectivity ones, we need to consider $\mathcal{A}^{\text {Llnj }}$ as a subcategory of $X \rightarrow$ which is not necessarily full. In the same vein, we define categories of lax fractions for subcategories $\Sigma$ of $X \rightarrow$.

Definition 3.1. Let $X$ be a category and $\Sigma$ a subcategory of the arrow category $X \rightarrow$. A category of lax fractions of $X$ for $\Sigma$ consists of a (quasi)category $X\left[\Sigma_{*}\right]$ and a functor $P_{\Sigma}: X \rightarrow X\left[\Sigma_{*}\right]$ such that:

(i) $P_{\Sigma} h$ is a left adjoint section, for every object $h$ of $\Sigma$.

(ii) For every morphism $(u, v): h \rightarrow h^{\prime}$ in $\Sigma, P_{\Sigma} u \cdot\left(P_{\Sigma} h\right)_{*}=\left(P_{\Sigma} h^{\prime}\right)_{*} \cdot P_{\Sigma} v$.

(iii) If $G: \mathcal{X} \rightarrow \mathcal{C}$ is another functor enjoying the properties (i) and (ii), then there is a unique functor $H: X\left[\Sigma_{*}\right] \rightarrow \mathcal{C}$ such that $H P_{\Sigma}=G$.

Remark 3.2. If we think of an ordinary category $X$ as an order-enriched one via the discrete order, i.e., the order $=$, then (ii) trivially holds, and Definition 3.1 becomes the usual definition of category of fractions.

Definition 3.3. Given a subcategory $\mathcal{A}$ of $\mathcal{X}$, we will denote by

$$
\mathcal{A} \text { LInj }
$$

the subcategory of the arrow category $\mathcal{X} \rightarrow$ consisting of:

(i) Objects: all morphisms $h$ of $\mathcal{X}$ such that all objects and morphisms of $\mathcal{A}$ are left-Kan injective with respect to $h$. That is, the class of objects of $\mathcal{A}^{\text {Llnj }}$ is $\mathcal{A}^{\text {Llnj }}$.

(ii) Morphisms: those morphisms $(u, v):(X \stackrel{h}{\rightarrow} Y) \rightarrow\left(X^{\prime} \stackrel{h^{\prime}}{\rightarrow} Y^{\prime}\right)$, with $h$ and $h^{\prime}$ in $\mathcal{A}$ Llnj, such that, for every $g: X^{\prime} \rightarrow A$, with $A \in \mathcal{A}$, we have that $(g u) / h=\left(g / h^{\prime}\right) v$ :

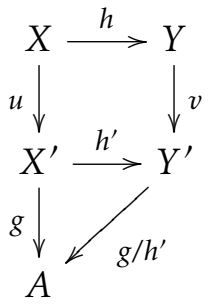


In other words, a morphism $(u, v):(X \stackrel{h}{\rightarrow} Y) \rightarrow\left(X^{\prime} \stackrel{h^{\prime}}{\rightarrow} Y^{\prime}\right)$ of $X \rightarrow$ is a morphism of $\mathcal{A} \stackrel{\text { Llnj }}{\text { iff it satisfies }}$ the equality $X(h, A)^{*} \cdot X(u, A)=X(v, A) \cdot X\left(h^{\prime}, A\right)^{*}$ for all objects $A \in \mathcal{A}$.

The next lemmas are going to be used in the proof of the main result of this section, Theorem 3.7.

Lemma 3.4. Let $\mathcal{A}$ be a KZ-reflective subcategory of $X$ with reflector functor $F: X \rightarrow \mathcal{A}$. Then, for every morphism $h: X \rightarrow Y$ in $X$ and every morphism $(u, v): h \rightarrow h^{\prime}$ in $X \rightarrow$, we have that:

(i) $h \in \mathcal{A}^{\text {LInj }}$ iff $F h$ is a left adjoint section in $\mathcal{A}$; and

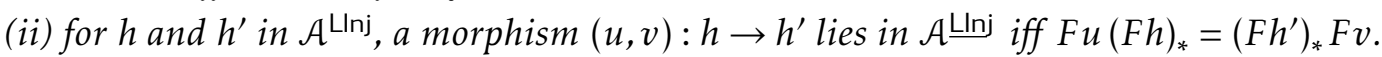

Proof. (i) was proved in [8] (see the last paragraph of 2.2).

(ii) It is easy to verify, and it was observed in [12], that, under the present conditions, given $a: X \rightarrow A$ with $A \in \mathcal{A}$, we have that

$$
a / h=\varepsilon_{A} \cdot F a \cdot(F h)_{*} \cdot \eta_{Y},
$$

where $\eta$ and $\varepsilon$ are the corresponding unit and counit. Let $(u, v): h \rightarrow h^{\prime}$ be a morphism of $\mathcal{A}$ ㄴǌ :

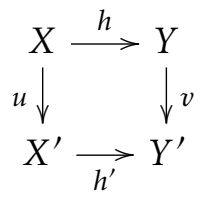

Then, for $\eta_{X^{\prime}}: X^{\prime} \rightarrow F X^{\prime}$, we have $\left(\eta_{X^{\prime}} / h^{\prime}\right) v=\left(\eta_{X^{\prime}} u\right) / h$, that is, by (2), $\varepsilon_{F X^{\prime}} F \eta_{X^{\prime}}\left(F h^{\prime}\right)_{*} \eta_{Y^{\prime}} v=$ $\varepsilon_{F X^{\prime}} F\left(\eta_{X^{\prime}} u\right)(F h)_{*} \eta_{Y}$. Consequently, $\left(F h^{\prime}\right)_{*} \eta_{Y^{\prime}} v=F u(F h)_{*} \eta_{Y}$, i.e., $\left(F h^{\prime}\right)_{*} F v \eta_{Y}=F u(F h)_{*} \eta_{Y}$; thus, $\left(F h^{\prime}\right)_{*} F v=F u(F h)_{*}$, since from (i) we know that $\left(F h^{\prime}\right)_{*} F v$ and $F u(F h)_{*}$ are both morphisms of $\mathcal{A}$.

Conversely, if the equality $\left(F h^{\prime}\right)_{*} F v=F u(F h)_{*}$ holds, for $d: X^{\prime} \rightarrow D$, with $D \in \mathcal{A}$, we have that $\left(d / h^{\prime}\right) v=\varepsilon_{D} F d\left(F h^{\prime}\right)_{*} \eta_{Y}, v=\varepsilon_{D} F d\left(F h^{\prime}\right)_{*} F v \eta_{Y}=\varepsilon_{D} F d F u(F h)_{*} \eta_{Y}=\varepsilon_{D} F(d u)(F h)_{*} \eta_{Y}=(d u) / h$.

Remark 3.5. ([8]) Let $\mathcal{A}$ be a reflective subcategory of $X$, with reflector functor $F$, unit $\eta$ and counit $\varepsilon$. Then $\mathcal{A}$ is KZ-reflective if and only if $F \varepsilon_{A} \geq \varepsilon_{F A}, A \in \mathcal{A}$, if and only if $\eta_{A} \varepsilon_{A} \geq \mathrm{id}_{F A}, A \in \mathcal{A}$. Then, when $\mathcal{A}$ is KZ-reflective, $\varepsilon_{A}$ is a left adjoint retraction, with $\left(\varepsilon_{A}\right)_{*}=\eta_{A}$. Moreover, every $F \eta_{X}$ is a left adjoint section, with $\left(F \eta_{X}\right)_{*}=\varepsilon_{F X}$. Thus, $\varepsilon_{F X}$ is simultaneously a right adjoint and a left adjoint satisfying the inequalities $F \eta_{X} \varepsilon_{F X} \leq \mathrm{id}_{F^{2} X} \leq \eta_{F X} \varepsilon_{F X}$.

Lemma 3.6. Let $\mathcal{A}$ be a KZ-reflective subcategory of $X$, with reflector $F$ and unit $\eta$. Then, for every $f: X \rightarrow Y,(f, F f): \eta_{X} \rightarrow \eta_{Y}$ is a morphism of the category $\mathcal{A}$ Llnj.

Proof. Indeed, with respect to the commutative square

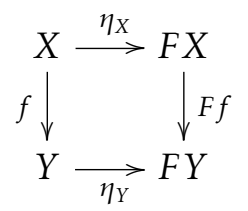

using Remark 3.5, we have that $F f\left(F \eta_{X}\right)_{*}=F f \varepsilon_{F X}=\varepsilon_{F Y} F^{2} f=\left(F \eta_{Y}\right)_{*} F^{2} f$; hence, by Lemma 3.4, the morphism $(f, F f)$ lies in $\mathcal{A}$ 늠. 
Theorem 3.7. Let $\mathcal{A}$ be a KZ-reflective subcategory of $\mathcal{X}$ with reflector functor $F: \mathcal{X} \rightarrow \mathcal{A}$. Then there exists a category $X\left[\Sigma_{*}\right]$ and a functor $P_{\Sigma}: X \rightarrow X\left[\Sigma_{*}\right]$ forming a category of lax fractions of $X$ for $\Sigma=\mathcal{A}$ 늠. Moreover, if $H: X\left[\Sigma_{*}\right] \rightarrow \mathcal{A}$ is the unique functor with $H P_{\Sigma}=F$, then for every $g: A \rightarrow B$ in $\mathcal{A}$ there is some $\bar{g}: X \rightarrow Y$ in $X\left[\Sigma_{*}\right]$ and a commutative diagram $H X \stackrel{H \bar{g}}{\rightarrow} H Y$ in $X$ with $r$ and $r^{\prime}$ left adjoint

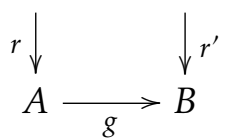

retractions.

Proof. Let $\eta$ and $\varepsilon$ be the corresponding unit and counit of the KZ-reflection of $\mathcal{X}$ into $\mathcal{A}$. Define a category $X\left[\Sigma_{*}\right]$ and a functor $P_{\Sigma}: X \rightarrow X\left[\Sigma_{*}\right]$ as follows:

- $\left|X\left[\Sigma_{*}\right]\right|=|X|$, where $|X|$ denotes the class of objects of $X$.

- for every $X, X^{\prime} \in|X|$, the poset $X\left[\Sigma_{*}\right]\left(X, X^{\prime}\right)$ is $\mathcal{A}\left(F X, F X^{\prime}\right)$;

- for every object $X$ of $X\left[\Sigma_{*}\right]\left(X, X^{\prime}\right)$ the identity $\operatorname{id}_{X}$ is just $\operatorname{id}_{F X}$, and the composition is defined as in $\mathcal{A}$;

- $P_{\Sigma} X=X$ and $P_{\Sigma} f=F f$, for every object $X$ and every morphism $f$ of $X$.

$X\left[\Sigma_{*}\right]$ is, up to isomorphism of categories, the Kleisli category of the monad induced in $X$ by $F$, and $P_{\Sigma}: X \rightarrow X\left[\Sigma_{*}\right]$ is the corresponding reflection of $X$ in it (cf. [19]). We show that $P_{\Sigma}: X \rightarrow X\left[\Sigma_{*}\right]$ is a category of lax fractions for $\mathcal{A}$ Llnj.

The satisfaction by $P_{\Sigma}$ of conditions (i) and (ii) of Definition 3.1 follows immediately from the definition of $P_{\Sigma}$ and Lemma 3.4.

Concerning (iii), let $G: X \rightarrow \mathcal{C}$ be a functor satisfying conditions (i) and (ii) of Definition 3.1. We want to define a functor $H: X\left[\Sigma_{*}\right] \rightarrow \mathcal{C}$ such that $H P_{\Sigma}=G$ and show that there is a unique such functor $H$.

First observe that if this functor $H$ exists, then we have

$$
H X=H P_{\Sigma} X=G X \text {, }
$$

for every $X \in\left|X\left[\Sigma_{*}\right]\right|$; and, for every morphism $f$ of $X$ for which $(F f)_{*}$ exists,

$$
H\left((F f)_{*}\right)=(H F f)_{*}=(G f)_{*},
$$

since we are dealing with order-enriched functors, which preserve adjunctions and retractions. In particular (see Remark 3.5),

$$
H\left(\varepsilon_{F X}\right)=H\left(\left(F \eta_{X}\right)_{*}\right)=\left(G \eta_{X}\right)_{*} .
$$

Moreover, given $f \in X\left[\Sigma_{*}\right]\left(X, X^{\prime}\right)$, i.e., $f: F X \rightarrow F X^{\prime}$ in $\mathcal{A}$, we have that $H f=H\left(f \varepsilon_{F X} F \eta_{X}\right)=$ $H\left(\varepsilon_{F X^{\prime}} \cdot F f \cdot F \eta_{X}\right)$; then, by $(5)$,

$$
H f=\left(G \eta_{X^{\prime}}\right)_{*} \cdot G f \cdot G\left(\eta_{X}\right) .
$$

The satisfaction of (3) and (6) defines $H$ uniquely, and the equality $H P_{\Sigma}=G$ is easily verified.

It remains to show that $H$ is indeed a functor. The preservation of identities is clear. To prove that $H$ preserves composition, let $f: F X \rightarrow F Y$ and $g: F Y \rightarrow F Z$ be two morphisms of $X\left[\Sigma_{*}\right](X, Y)$ and $X\left[\sum_{*}\right](Y, Z)$, respectively. We want to show that $H(g f)=H g \cdot H f$.

Due to the equality $\left(F \eta_{X}\right)_{*}=\varepsilon_{F X}$, given in Remark 3.5, we have that, for every morphism $f$ : $F X \rightarrow F Y$ of $\mathcal{A}, f=\left(F \eta_{Y}\right)_{*} \cdot F f \cdot F\left(\eta_{X}\right)$. Taking this into account and the fact that $G$ preserves 
adjunctions, we have:

$$
G g G f=\left(G F \eta_{Z}\right)_{*} \cdot G F g \cdot G F\left(\eta_{Y}\right) \cdot\left(G F \eta_{Y}\right)_{*} \cdot G F f \cdot G F\left(\eta_{X}\right) .
$$

Composing with $\left(G \eta_{Z}\right)_{*}$ on the left-hand side and with $G \eta_{X}$ on the right-hand side, and using (6), we obtain:

$$
H(g f)=\left(G \eta_{Z}\right)_{*} \cdot\left(G F \eta_{Z}\right)_{*} \cdot G F g \cdot G F\left(\eta_{Y}\right) \cdot\left(G F \eta_{Y}\right)_{*} \cdot G F f \cdot G F\left(\eta_{X}\right) \cdot G \eta_{X} \cdot
$$

But the diagram

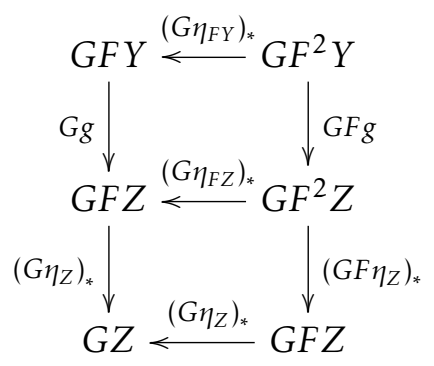

is commutative: the top square commutes, because $(g, F g): \eta_{F Y} \rightarrow \eta_{F Z}$ is a morphism of $\Sigma=$ $\mathcal{A}$ Llnj, by Lemma 3.6, and $G$ satisfies condition (ii) of Definition 3.1; the bottom square commutes because all morphisms $\eta_{Z}, F \eta_{Z}$ and $\eta_{F Z}$ belong to $\Sigma$, thus $\left(G \eta_{Z}\right)_{*},\left(G F \eta_{Z}\right)_{*}$ and $\left(G \eta_{F Z}\right)_{*}$ are defined and, from the equality $F \eta_{Z} \cdot \eta_{Z}=\eta_{F Z} \cdot \eta_{Z}$, it follows the required equality. Consequently, we have:

$$
\left(G \eta_{Z}\right)_{*} \cdot\left(G F \eta_{Z}\right)_{*} \cdot G F g=\left(G \eta_{Z}\right)_{*} \cdot G g \cdot\left(G \eta_{F Y}\right)_{*} .
$$

Moreover,

$$
G F f \cdot G F\left(\eta_{X}\right) \cdot G \eta_{X}=G F f \cdot G \eta_{F X} \cdot G \eta_{X}=G\left(\eta_{F Y}\right) \cdot G f \cdot G \eta_{X} \cdot
$$

Therefore, by applying (8) and (9) to the right-hand side of (7), we get

$$
H(g f)=\left(G \eta_{Z}\right)_{*} \cdot G g \cdot\left(G \eta_{F Y}\right)_{*} \cdot G F\left(\eta_{Y}\right) \cdot\left(G F \eta_{Y}\right)_{*} \cdot G\left(\eta_{F Y}\right) \cdot G f \cdot G \eta_{X} \cdot
$$

In order to conclude that the right-hand side of the last equality is precisely

$$
H g \cdot H f=\left(G\left(\eta_{Z}\right)\right)_{*} \cdot G(g) \cdot G\left(\eta_{Y}\right) \cdot\left(G\left(\eta_{Y}\right)\right)_{*} \cdot G f \cdot G\left(\eta_{X}\right),
$$

it suffices to show that $\left(G \eta_{F Y}\right)_{*} \cdot G F\left(\eta_{Y}\right) \cdot\left(G F \eta_{Y}\right)_{*} \cdot G\left(\eta_{F Y}\right)=G\left(\eta_{Y}\right)\left(G\left(\eta_{Y}\right)\right)_{*}$. This is easy:

$$
\begin{aligned}
\left(G \eta_{F Y}\right)_{*} \cdot G F\left(\eta_{Y}\right) \cdot\left(G F \eta_{Y}\right)_{*} \cdot G\left(\eta_{F Y}\right) & =G \eta_{Y} \cdot\left(G \eta_{Y}\right)_{*} \cdot\left(G F \eta_{Y}\right)_{*} \cdot G\left(\eta_{F Y}\right), \quad \text { by using Lemma } 3.6 \\
& =G \eta_{Y} \cdot\left(G \eta_{Y}\right)_{*} \cdot \operatorname{id} d_{F Y} \\
& =G \eta_{Y} \cdot\left(G \eta_{Y}\right)_{*} .
\end{aligned}
$$

The order-enrichment of $H$ is immediate from the definition of $H$, since $G$ is so.

Finally, from Lemma 3.4, we know that the reflector functor $F: X \rightarrow \mathcal{A}$ satisfies conditions (i) and (ii). Thus, as we have just seen, the unique functor $H: X\left[\Sigma_{*}\right] \rightarrow \mathcal{A}$ such that $H P_{\Sigma}=F$ is defined by $H X=F X$ and $H f=\left(F \eta_{Y}\right)_{*} \cdot F f \cdot F \eta_{X}=\varepsilon_{F Y} \cdot F f \cdot F \eta_{X}=f \cdot \varepsilon_{F X} \cdot F \eta_{X}=f$. For every morphism $g: A \rightarrow B$ of $\mathcal{A}$, we have $F g \in X\left[\sum_{*}\right](A, B)$, with $H(F g)=(F g: F A \rightarrow F B)$, and thus we have a commutative diagram of the form

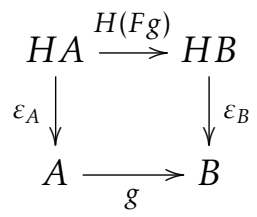


with $\varepsilon_{A}$ and $\varepsilon_{B}$ left adjoint retractions in $X$ (see Remark 3.5).

Remark 3.8. Under the conditions of the above theorem, let $E: \mathcal{A} \rightarrow X$ be the corresponding inclusion functor and put $K=P_{\Sigma} E: \mathcal{A} \rightarrow X\left[\Sigma_{*}\right]$. Then $K$ is faithful, because, for every morphism $f: A \rightarrow A^{\prime}$ of $\mathcal{A}$, we have that $f=\varepsilon_{A^{\prime}} F f \eta_{A}$. And it has the property that, for every morphism $g: X \rightarrow X^{\prime}$ in $X\left[\Sigma_{*}\right]$, there are a morphism $f: A \rightarrow A^{\prime}$ in $\mathcal{A}$ and a commutative diagram

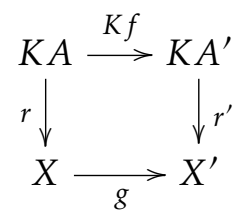

in $X\left[\sum_{*}\right]$ with $r$ and $r^{\prime}$ retractions which are simultaneously left and right adjoints. Indeed, it suffices to take $r=\varepsilon_{F X}$ and $r^{\prime}=\varepsilon_{F X^{\prime}}$ (see Remark 3.5).

Remark 3.9. As observed before, the category $X\left[\Sigma_{*}\right]$ described in the proof of the above theorem is the Kleisli category for the monad over $\mathcal{X}$ induced by its KZ-reflection into $\mathcal{A}$. We point out that in [14] the authors show that, for every monad, the Kleisli category can always be seen as a category of (generalized) fractions.

\section{A left calculus of lax fractions}

In this section we introduce the notion of a left calculus of lax fractions relatively to a subcategory $\Sigma$ of the arrow category $X \rightarrow$, which generalizes the usual left calculus of fractions ([13]) and allows us to describe the category of lax fractions of $\mathcal{X}$ for $\sum$ in terms of formal fractions $s_{*} f$ represented by cospans $\bullet \stackrel{f}{\longrightarrow} \bullet \stackrel{s}{\longleftarrow} \bullet$ with $s$ an object of $\Sigma$.

$\sum$-squares, as described next, are going to be used to define and manipulate the left calculus of lax fractions.

Terminology 4.1. Given a subcategory $\Sigma$ of $X^{\rightarrow}$, we use a square of the form

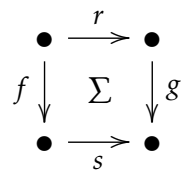

to indicate that $f, g, r$ and $s$ are morphisms of $X$ such that $(f, g): r \rightarrow s$ is a morphism of $\Sigma$, and a square of this type is called a $\sum$-square.

Moreover, by a a $\sum$-span we mean a span $\bullet \stackrel{r}{\longleftarrow} \bullet \stackrel{f}{\longrightarrow} \bullet$ with $r$ an object of $\Sigma$. And a $\Sigma$ cospan from $A$ to $B$ is a cospan $A \stackrel{g}{\longrightarrow} J<^{s} B$ with $s$ an object of $\Sigma$.

When we have $(r, f)$ and $(g, s)$ forming a $\sum$-square as above, we say that the $\sum$-span $(r, f)$ covers the $\sum$-cospan $(g, s)$.

Thinking of a $\sum$-span $\bullet \stackrel{r}{\longleftarrow} \bullet \stackrel{f}{\longrightarrow} \bullet$ as a formal representation of the (lax) fraction $f r_{*}$, and of the $\sum$-cospan $\bullet \stackrel{g}{\longrightarrow} \bullet \stackrel{s}{<}$ as a formal representation of the (lax) fraction $s_{*} g$, the above $\Sigma$-square represents the formal equality $f r_{*}=s_{*} g$.

Definition 4.2. A subcategory $\Sigma$ of $X \rightarrow$ is said to admit a left calculus of lax fractions of $X$ if it satisfies the following conditions: 
1. Identity. The identities of $X$ are objects of $\Sigma$ and $\stackrel{\text { id }}{\rightarrow} \bullet$ for all objects $s$ of $\Sigma$.

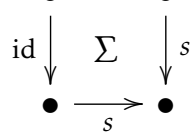

2. Composition. If we have

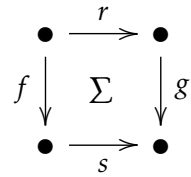

and
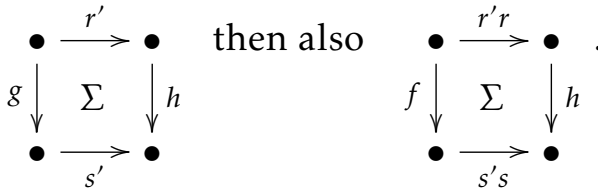

3. Square. For every $\sum$-span $\bullet \stackrel{r}{\longleftarrow} \stackrel{f}{\longrightarrow} \bullet$, there are morphisms $r^{\prime}$ and $f^{\prime}$ such that

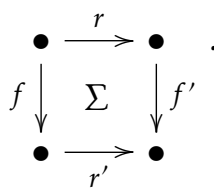

4. Coinsertion. Given a diagram $\stackrel{r}{\longrightarrow} \bullet \quad$ where the inner square is a $\sum$-square, and such

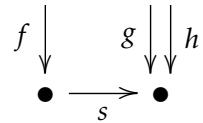

that $g r \leq h r$, then there is a morphism $t$, whose domain is the codomain of $s$, satisfying the following conditions:

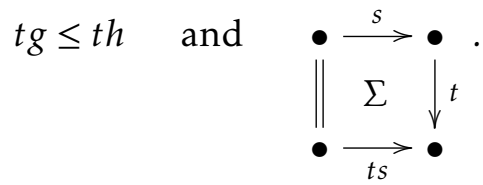

Remark 4.3. Combining the composition of morphisms in the category $\Sigma$ with the one given by Composition, we have that any square obtained by finite horizontal and vertical compositions of $\sum$-squares is a $\sum$-square. This is going to be very useful in the proofs of this section.

Examples 4.4. 1. Recall that a class of morphisms $\Sigma$ of an ordinary category $X$ admits a left calculus of fractions if it satisfies the following conditions:

1 '. $\Sigma$ contains all identities of $X$.

$2^{\prime}$. $\sum$ is closed under composition.

3'. For every span $\bullet<\frac{r}{<} \bullet \stackrel{f}{\longrightarrow} \bullet$ with $r \in \Sigma$, there is a cospan $\bullet \stackrel{f^{\prime}}{\longrightarrow} \bullet<\frac{r^{\prime}}{<} \bullet$ with $r^{\prime} \in \Sigma$ and $f^{\prime} r=r^{\prime} f$.

4'. If we have a diagram $\bullet \stackrel{r}{\longrightarrow} \bullet \stackrel{h}{\underset{g}{\longrightarrow}} \bullet$ with $r \in \Sigma$ and $g r=h r$ then there is some $t \in \Sigma$ with $\operatorname{tg}=t h$

Let $X$ be an ordinary category, equivalently, a category enriched with the discrete order $=$. Let $\Sigma$ be a class of morphisms of $X$, regarded as a full subcategory of $X \rightarrow$. Then $\Sigma$ admits a left calculus of lax fractions if and only if it admits a left calculus of fractions in the usual sense. Indeed, the equivalence of the three first conditions is immediately seen. To show that, in the presence of 1-3, 4 implies 4 ', let $g$ and $h$ be a pair of morphisms equalized by a morphism $r$ of $\sum$. For $f=g r=h r$ and $s=$ id we obtain a diagram as the first one in Definition 4.2.4, which is a $\sum$-square because of the fullness of $\Sigma$. Consequently, there is some morphism $t$ under the conditions of the second diagram of Definition 4.2.4; since $s$ is the identity, we conclude that $t \in \Sigma$. Conversely, given a 
diagram as the first one in Definition 4.2.4, with $g r=h r$, let $t$ be a morphism of $\sum$ such that $t g=t h$. Then, the second diagram of Definition 4.2.4 is indeed a $\sum$-square, since $t s \in \Sigma$.

In this case $P_{\Sigma}: X \rightarrow X\left[\Sigma_{*}\right]$ is just the category of fractions $P_{\Sigma}: X \rightarrow X\left[\Sigma^{-1}\right]$. Moreover, (i) every map of $X\left[\Sigma^{-1}\right]$ can be represented as $\left(P_{\Sigma} s\right)^{-1} P_{\Sigma} f$ with $s \in \Sigma$, and (ii) $\left(P_{\Sigma} s\right)^{-1} P_{\Sigma} f=\left(P_{\Sigma} t\right)^{-1} P_{\Sigma} g$ iff there is a commutative diagram in $X$ of the form

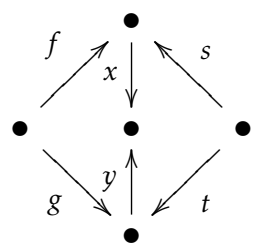

with $x s=y t$ in $\Sigma$. In [7], J. Bénabou presents a calculus of fractions which provides necessary and sufficient conditions on $\sum$ for (i) and (ii).

2. Let $\Sigma$ be the subcategory of $X \rightarrow$ whose objects are all left adjoint sections of $X$, and the morphisms between them are all $(f, g): r \rightarrow s$ with $f r_{*}=s_{*} g$. Then $\sum$ is clearly a subcategory of $X$, and it admits a left calculus of lax fractions. To show Coinsertion, given a morphism $(f, g): r \rightarrow s$, let $h$ be a morphism of $X$ with $g r \leq h r$; then $s_{*}$ plays the role of $t$ in Definition 4.2 , the inequality being obtained as follows: $s_{*} g=s_{*} s f r_{*}=s_{*} g r r_{*} \leq s_{*} h r r_{*} \leq s_{*} h$.

3. Let $X$ be an order-enriched category with conical pushouts (see Section 5). A morphism $e$ : $X \rightarrow Y$ of $X$ is said to be order-epic if, for every pair of morphisms $f, g: Y \rightarrow Z$ with $f e \leq g e$, we have that $f \leq g$. It is easily seen that every (conical) pushout of an order-epic morphism along an arbitrary morphism is also order-epic. Let $\Sigma$ be the subcategory of $\mathcal{X} \rightarrow$ defined as follows. The objects are all order-epic morphisms, and the morphisms are all morphisms of $X^{\rightarrow}$ of the form (id, $e):$ id $\rightarrow e$ with $e$ order-epic, represented by the square $\bullet=\bullet$, all morphisms $(f, g): e \rightarrow e^{\prime}$

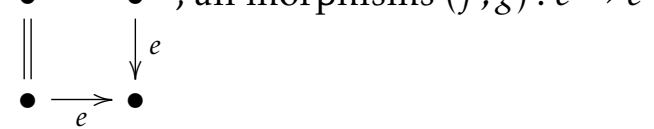

of $X$ such that the square $\stackrel{e}{\longrightarrow} \bullet$ is a pushout, and all morphisms of $X \rightarrow$ obtained by finite

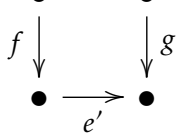

horizontal and vertical composition of these two types of squares. It is easy to see that $\sum$ is indeed a subcategory of $X \rightarrow$ which admits a left calculus of lax fractions.

4. In the category Pos, we say that a morphism $m: X \rightarrow Y$ is an (order) embedding if it satisfies the condition $m(x) \leq m\left(x^{\prime}\right) \Rightarrow x \leq x^{\prime}$, for all $x, x^{\prime} \in X$. We know that, in Pos, every complete lattice is Kan-injective with respect to embeddings, and given $f: X \rightarrow C$ with $C$ a complete lattice $f / m$ is defined by (see [6] and [1])

$$
(f / m)(b)=\bigvee_{m(x) \leq b} f(x) .
$$

Moreover, embeddings are precisely those morphisms $m: X \rightarrow Y$ with respect to which the twoelement chain $D=(0<1)$ is Kan-injective; indeed, given $a$, $a^{\prime} \in X$ with $m(a) \leq m\left(a^{\prime}\right)$, define $f: X \rightarrow$ $D$ by $f(x)=1$ if $a \leq x$, otherwise $f(x)=0$. Then, if $D$ is Kan-injective with respect to $m$, we have $1=f(a)=(f / m) m(a) \leq(f / m) m\left(a^{\prime}\right)=f\left(a^{\prime}\right)$, and this implies the equality $f\left(a^{\prime}\right)=1$, i.e. $a \leq a^{\prime}$.

Let $\sum$ be the subcategory of Pos $\rightarrow$ consisting of: 
- Objects: all embeddings;

- Morphisms: all morphism $(u, v): m \rightarrow n$, with $m: X \rightarrow Y$ and $n: Z \rightarrow W$ embeddings, satisfying the following condition, for all $y \in Y$ and $z \in Z$ :

$$
n(z) \leq v(y) \Longrightarrow \text { there is some } x \in X \text { with } z \leq u(x) \text { and } m(x) \leq y .
$$

We show that $\Sigma=D$ LInj. As a consequence, $\Sigma$ admits a left calculus of lax fractions. Indeed, in Proposition 5.3 we will see that if $X$ has finite weighted colimits then, for every subcategory $\mathcal{A}$ of $X, \Sigma=\mathcal{A} \underline{\text { Lnj }}$ always admits a left calculus of fractions.

Since we already have seen that embeddings are precisely the morphisms of $X$ with respect to which $D$ is Kan-injective, it remains to show that (11) characterizes the morphisms of $D$ Llmj. Let then the morphism $(u, v): m \rightarrow n$ of Pos $\rightarrow$ satisfy (11), and consider a morphism $f: Z \rightarrow D$. We want to show that $(f u) / m=(f / n) v$. Since $(f u) / m \leq(f / n) v$ always holds, it suffices to show that, for each $y \in Y,((f / n) v)(y)=1$ implies $((f u) / m)(y)=1$; in other words, taking into account (10), if $y \in Y$ and $z \in Z$ are such that $f(z)=1$ and $n(z) \leq v(y)$, then there is some $x \in X$ with $f u(x)=1$ and $m(x) \leq y$. But the satisfaction of this last condition is clearly ensured by (11). Conversely, let

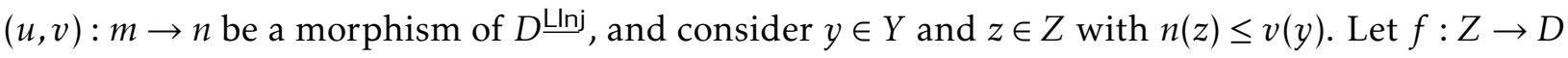
be defined by $f\left(z^{\prime}\right)=1$ if $z \leq z^{\prime}$, otherwise, $f\left(z^{\prime}\right)=0$. Since $f(z)=1$ and $n(z) \leq v(y)$, we have that $((f / n) v)(y)=1$. Thus also $((f u) / m)(y)=1$. But this means that there is some $x \in X$ with $m(x) \leq y$ and $(f u)(x)=1$, the last equality meaning that $z \leq u(x)$.

Let $\Omega_{0}$ be the contravariant endofunctor of Pos sending every poset $X$ to the poset $\Omega_{0} X$ of its lower sets, and every monotone map $f: X \rightarrow Y$ to the preimage map $\Omega_{0} f: \Omega_{0} Y \rightarrow \Omega_{0} X$. In [2], we show that condition (11) above is equivalent to the Beck-Chevalley condition $\left(\Omega_{0} u\right)^{*} \cdot \Omega_{0} m=$ $\Omega_{0} n \cdot\left(\Omega_{0} b\right)^{*}$, where $-{ }^{*}$ stands for the left adjoint.

5. (cf. [2]) Let Loc be the category of locales (i.e., frames) and localic maps, i.e., maps $f$ preserving all infima and whose left adjoint $f^{*}$ preserves finite meets. Recall that embeddings in Loc are precisely the localic maps $h$ made split monomorphisms by its left adjoint: $h^{*} h=\mathrm{id}([15])$.

Let $\Sigma_{0}$ be the subcategory of Loc $\rightarrow$ having all embeddings as objects and whose morphisms are those $(u, v): m \rightarrow n$ of $\operatorname{Loc}^{\rightarrow}$ satisfying the Beck-Chevalley condition $v^{*} n=m u^{*}$. We are going to show that $\Sigma_{0}$ admits a left calculus of lax fractions.

In [9] we showed that for every finitely generated frame $F$, given an embedding $m: X \rightarrow Y$ and $f: X \rightarrow F$, the map $m f^{*}$ is a frame homomorphism, thus $\left(m f^{*}\right)_{*}$ is localic, and moreover

$$
f / m=\left(m f^{*}\right)_{*} .
$$

We also proved that embeddings are precisely the localic maps with respect to which the free frame $F_{1}$ generated by $1=\{0\}$ is Kan-injective. In order to conclude that $\Sigma_{0}$ admits a left calculus of lax fractions we show that $\Sigma_{0}=F_{1}^{\operatorname{Lnj}}$. Then, since Loc has finite weighted colimits, the result follows from Proposition 5.3.

Indeed, assume that in the commutative square

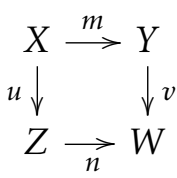


$m$ and $n$ are embeddings and $m u^{*}=v^{*} n$. Then, for every $f: Z \rightarrow F_{1}$, we have:

$$
(f / n) v=\left(n f^{*}\right)_{*} v=\left(v^{*}\left(n f^{*}\right)\right)_{*}=\left(m u^{*} f^{*}\right)_{*}=\left(m(f u)^{*}\right)_{*}=(f u) / m .
$$

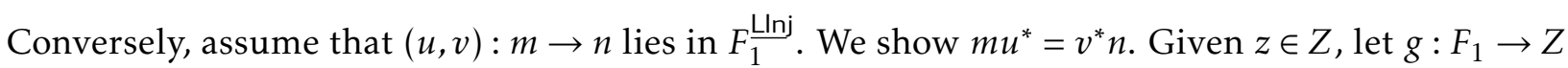
be the frame homomorphism sending the element 0 to $z$. The localic map $g_{*}: Z \rightarrow F_{1}$ satisfies the equality $\left(g_{*} / n\right) v=\left(g_{*} u\right) / m$, i.e., by $(12),(n g)_{*} v=\left(m u^{*} g\right)_{*}$; then, by applying the operator $-^{*}$ to the last equality, we obtain $v^{*} n g=m u^{*} g$, thus $v^{*} n(z)=v^{*} n g(0)=m u^{*} g(0)=m u^{*}(z)$.

6. Recall that in Loc dense embeddings are those preserving the bottom $\perp$, and flat embeddings are those preserving finite joins. Let now $F_{0}, F_{1}$ and $F_{2}$ be the free frames generated by the empty set, $1=\{0\}$ and $2=\{0,1\}$, respectively, and let $f_{i}: F_{i} \rightarrow F_{1}, i=0,2$, be the localic maps determined by $f_{0}(\perp)=0, f_{2}(0 \vee 1)=0$ and $f_{2}(x)=\perp$ for $x \neq \top, 0 \vee 1$. In [9] dense embeddings were characterized as precisely the localic maps with respect to which the morphism $f_{0}$ is Kan-injective. And flat embeddings were characterized there as precisely those morphisms with respect to which both $f_{0}$ and $f_{2}$ are Kan-injective. Let $\Sigma_{1}$ and $\Sigma_{2}$ be the full subcategories of the category $\Sigma_{0}=F_{1}^{\text {Llnj }}$, described in 5, consisting of all dense embeddings, and all flat embeddings, respectively. Both $\Sigma_{1}$ and $\Sigma_{2}$ admit a left calculus of lax fractions. Indeed, by using the same arguments as in the previous example, we see that $\Sigma_{1}=\left\{f_{0}\right\}^{\text {LInj }}$ and $\Sigma_{2}=\left\{f_{0}, f_{2}\right\}$ LInj .

7. Let $\mathrm{Top}_{0}$ be the category of $T_{0}$-topological spaces and continuous maps, considered as an orderenriched category via the dual of the specialization order. Let Lc : Top $_{0} \rightarrow$ Loc be the functor taking every space $X$ to the frame of its open sets $\Omega X$, and every continuous map $f: X \rightarrow Y$ to the right adjoint of the preimage map $f^{-1}: \Omega Y \rightarrow \Omega X$. Then the subcategory $\Sigma$ of $\operatorname{Top}_{0} \vec{x}$ consisting of all (topological) embeddings and all morphisms $(u, v): m \rightarrow n$ between embeddings such that $(\operatorname{Lc}(u), \operatorname{Lc}(v)): \operatorname{Lc}(m) \rightarrow \operatorname{Lc}(n)$ belongs to the category $\Sigma_{0}$ described above (in 5) admits

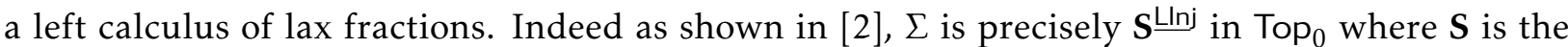
Sierpiński space.

A collection of examples of subcategories $\Sigma=\mathcal{A}$ Llnj of $X \rightarrow$ admitting a left calculus of lax fractions (which indeed includes Examples 3, 5 and 6 of 4.4 (see [9]), is obtained from the next proposition.

Proposition 4.5. If $\mathcal{A}$ is a KZ-reflective subcategory of $X$, then $\Sigma=\mathcal{A} \underline{\text { LInj }}$ admits a left calculus of lax fractions.

Proof. Using Lemma 3.4, the satisfaction of Identity and Composition is clear. To obtain Square, in 4.2.3 let $X$ be the domain of $r$ and let $Y$ and $Z$ be the codomains of $r$ and $f$, respectively; put $r^{\prime}=\eta_{Z}$ and $f^{\prime}=F f(F r)_{*} \eta_{Y}$. From Remark 3.5, we know that $\left(F \eta_{Z}\right)_{*}=\varepsilon_{F Z}$, and then, since $F(F r)_{*} \cdot F \eta_{Y} \cdot \eta_{Y}=F(F r)_{*} \cdot \eta_{F Y} \cdot \eta_{Y}=\eta_{F X} \cdot(F r)_{*} \cdot \eta_{Y}$, we have that

\section{$\left(F \eta_{Z}\right)_{*} \cdot F^{2} f \cdot F(F r)_{*} \cdot F \eta_{Y} \cdot \eta_{Y}=F f \cdot \varepsilon_{F X} \cdot \eta_{F X} \cdot(F r)_{*} \cdot \eta_{Y}=F f \cdot(F r)_{*} \cdot \eta_{Y} \cdot$}

Since $\left(F \eta_{Z}\right)_{*} \cdot F\left(F f \cdot(F r)_{*} \cdot \eta_{Y}\right)$ and $F f \cdot(F r)_{*}$ are both morphisms of $\mathcal{A}$ (see 2.2), we conclude that they are equal; that is, by Lemma 3.4 again, our square is of $\sum$ type.

To show Coinsertion, let us have a diagram $X \stackrel{r}{\longrightarrow} Y \quad$ where the inner square is a $\Sigma$-square

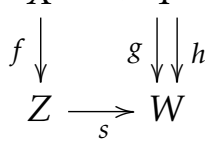

and with $g r \leq h r$. Put $t=(F s)_{*} \eta_{W}$. Then, $t g=(F s)_{*} \eta_{W} g=(F s)_{*} F g \eta_{Y}=F f(F r)_{*} \eta_{Y}=(F s)_{*} F s F f(F r)_{*} \eta_{Y}$. 
But $F s F f(F r)_{*}=F g F r(F r)_{*} \leq F h F r(F r)_{*} \leq F h$. Thus

$$
\operatorname{tg} \leq(F s)_{*} F h \eta_{Y}=(F s)_{*} \eta_{W} h=t h .
$$

Moreover, we have $t s=(F s)_{*} \eta_{W} s=(F s)_{*} F s \eta_{Y}=\eta_{Y}$; hence, by Lemma 3.4 and Remark 3.5, $t s \in \sum$. To show that (id, $t): s \rightarrow t s$ is a morphism of $\sum$ we also use property (ii) of Lemma 3.4: $(F(t s))_{*} F t=$ $\left(F \eta_{Y}\right)_{*} F(F s)_{*} F \eta_{W}=\varepsilon_{F Y} F(F s)_{*} F \eta_{W}=(F s)_{*} \varepsilon_{F W} F \eta_{W}=(F s)_{*}$.

Let $\Sigma$ be a subcategory of $X \rightarrow$ admitting a left calculus of lax fractions. We are going to see that then we obtain a category of lax fractions as follows: the objects of $X\left[\Sigma_{*}\right]$ are those of $X$, and the morphisms are going to be equivalence classes of $\Sigma$-cospans. In general, $X\left[\Sigma_{*}\right]$ is not locally small (even if $X$ is so), analogously to what happens in the ordinary case to $X\left[\Sigma^{-1}\right]$ for $\Sigma$ admitting a left calculus of fractions.

The following definitions and lemmas are a preparation for Theorem 4.11 below.

4.6. The relation $\leq$ between $\sum$-cospans. A $\sum$-cospan from $A$ to $B$ of the form

$$
A \stackrel{f}{\longrightarrow} I<s
$$

will be denoted by $(f, I, s)$ or just by $(f, s)$.

Given objects $A$ and $B$ of $X$, we consider a relation $\leq$ between $\sum$-cospans from $A$ to $B$ given by

$$
(f, I, s) \leq(g, J, t)
$$

if there is a diagram of the form

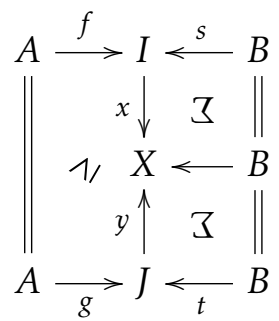

where, as indicated, $x f \leq y g$, and the two squares on the right-hand side are $\sum$-squares, i.e., (id, $x$ ): $s \rightarrow s x$ and (id, $y): t \rightarrow y t$ are morphisms of $\sum$ with $x s=y t$.

Lemma 4.7. For $\sum$ admitting a left calculus of lax fractions, let $A \stackrel{r}{\longleftarrow} D \stackrel{d}{\longrightarrow} B$ be a $\sum$-span covering the two $\Sigma$-cospans $A \stackrel{f_{i}}{\longrightarrow} I_{i} \stackrel{s_{i}}{\longleftarrow} B, i=1,2$ (see Terminology 4.1). Then $\left(f_{1}, I_{1}, s_{1}\right) \leq\left(f_{2}, I_{2}, s_{2}\right)$, and, analogously, $\left(f_{2}, I_{2}, s_{2}\right) \leq\left(f_{1}, I_{1}, s_{1}\right)$.

Proof. We show that $\left(f_{1}, I_{1}, s_{1}\right) \leq\left(f_{2}, I_{2}, s_{2}\right)$. Using Square, form the $\sum$-square

$$
\begin{gathered}
B \stackrel{s_{1}}{\longrightarrow} I_{1} . \\
s_{2} \downarrow \quad \sum \quad \downarrow^{r_{1}} \\
I_{2} \underset{r_{2}}{\longrightarrow} J
\end{gathered}
$$


Since, by hypothesis, $\left(d, f_{i}\right): r \rightarrow s_{i}$ is a morphism of $\sum$ for $i=1,2$, by vertical composition of $\sum$-squares, we obtain the $\sum$-square $\quad D \stackrel{r}{\longrightarrow} A$. Moreover, $\left(r_{1} f_{1}\right) r=r_{1} s_{1} d=r_{2} s_{2} d=\left(r_{2} f_{2}\right) r$.

$$
\begin{aligned}
& s_{2} d \downarrow \quad \Sigma \quad \downarrow r_{1} f_{1} \\
& I_{2} \underset{r_{2}}{\longrightarrow} J
\end{aligned}
$$

Consequently, by Coinsertion, there is some morphism $p: J \rightarrow I_{0}$ such that $p\left(r_{1} f_{1}\right) \leq p\left(r_{2} f_{2}\right)$, and

$$
\begin{aligned}
& B \stackrel{r_{2}}{\longrightarrow} J . \\
& \| \underset{p r_{2}}{\longrightarrow} I_{0}
\end{aligned} .
$$

To conclude that $\left(f_{1}, I_{1}, s_{1}\right) \leq\left(f_{2}, I_{2}, s_{2}\right)$, it remains to verify that the two squares on the right-hand side of the following diagram are of $\sum$ type: $A \stackrel{f_{1}}{\longrightarrow} I_{1} \stackrel{s_{1}}{\longleftarrow} B$. Concerning the bottom one, it

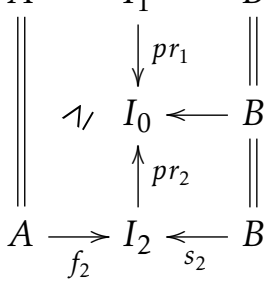

follows from the composition of the following $\sum$-squares, where we use (14), the fact that $\sum$ is a subcategory of $X^{\rightarrow}$, and Identity:

$$
\begin{aligned}
& B \stackrel{s_{2}}{\longrightarrow} I_{1}=I_{1} \\
& \| \begin{array}{c}
\Sigma \\
B \stackrel{s_{2}}{\longrightarrow} I_{1} \stackrel{r_{2}}{\longrightarrow} Z \\
\longrightarrow
\end{array} \\
& \| \Sigma \quad \Sigma \quad \Sigma \quad p \\
& B \underset{s_{2}}{\longrightarrow} X \underset{p r_{2}}{\longrightarrow} I_{0}
\end{aligned}
$$

Concerning the top one, observe that, from (13), Identity and Composition, we have that the outside square of the diagram $B=B \stackrel{s_{1}}{\longrightarrow} I_{1} \quad$ is a $\sum$ one. Now, composing vertically with the $\sum$-square

$$
\begin{aligned}
& \| s_{2} \downarrow \quad \Sigma \quad \downarrow^{r_{1}} \\
& B \underset{s_{2}}{\longrightarrow} I_{2} \underset{r_{2}}{\longrightarrow} J
\end{aligned}
$$

given by the composition of the two $\sum$-squares in the bottom of (15), and taking into account that $r_{2} s_{2}=r_{1} s_{1}$, we obtain the desired $\sum$-square.

Analogously, we can show that $\left(f_{2}, I_{2}, s_{2}\right) \leq\left(f_{1}, I_{1}, s_{1}\right)$.

Lemma 4.8. The relation $\leq$ on the class of all $\Sigma$-cospans is reflexive and transitive.

Proof. Reflexivity is clear, since $\stackrel{s}{\longrightarrow} \bullet$, because $\Sigma$ is a subcategory of $X^{\rightarrow}$ and (id,id) $: s \rightarrow s$ id $\underset{\bullet}{\stackrel{\Sigma}{\longrightarrow} \bullet} \stackrel{\downarrow \text { id }}{\longrightarrow}$

is the identity morphism on $s$. 
Concerning transitivity, let $(f, I, s),(g, J, t)$ and $(h, K, u)$ be $\Sigma$-cospans from $A$ to $B$ such that $(f, I, s) \leq(g, J, t)$ and $(g, J, t) \leq(h, K, u)$ through the following diagram:

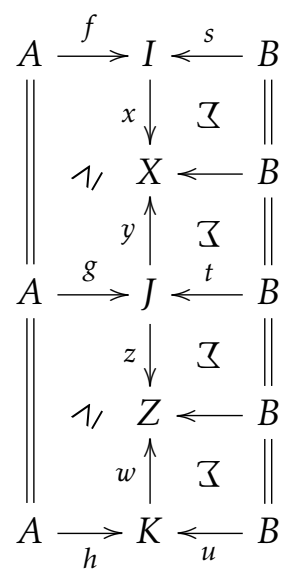

Then we have that the $\sum$-span $B \stackrel{\operatorname{id}_{B}}{\longleftarrow} B \stackrel{t}{\longrightarrow} J$ covers both the $\sum$-cospans $J \stackrel{y}{\longrightarrow} X \stackrel{y t}{\longleftarrow} B$ and $J \stackrel{z}{\longrightarrow} Z \stackrel{z t}{\longrightarrow} B$. Consequently, by Lemma 4.7, $(y, y t) \leq(z, z t)$. Therefore, there are morphisms $a: X \rightarrow Y$ and $b: Z \rightarrow Y$ with which we obtain the diagram

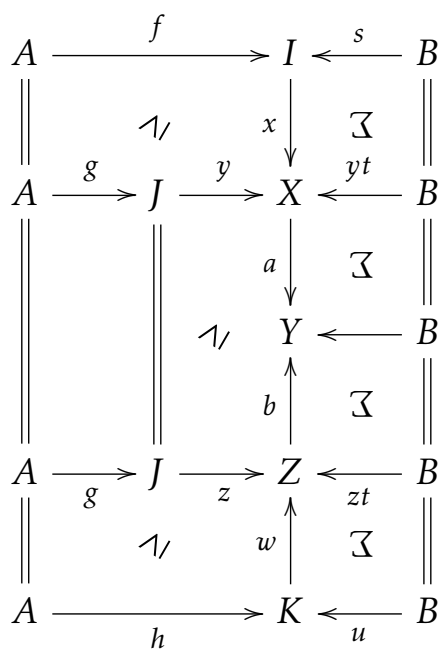

with $(a x) f \leq a y g \leq b z g \leq(b w) h$. Thus $(f, s) \leq(h, u)$.

4.9. The equivalence classes of $\sum$-cospans and their composition. We say that two $\sum$-cospans $(f, s)$ and $(g, t)$ with the same domain and codomain are equivalent, and write

$$
(f, s) \equiv(g, t)
$$

whenever $(f, s) \leq(g, t)$ and $(g, t) \leq(f, s)$.

Since $\leq$ is reflexive and transitive, $\equiv$ is an equivalence relation.

We denote the equivalence class of a $\sum$-cospan $(f, s)$ by $[(f, s)]$. When there is no reason for confusion, we also represent the equivalence class by one of its elements.

Since $\leq$ is reflexive and transitive, we obtain a partial order $\leq$ between equivalence classes of $\sum$-cospans with the same domain and codomain as follows:

$$
[(f, s)] \leq[(g, t)] \text { whenever }(f, s) \leq(g, t) \text {. }
$$


In particular, we conclude that, for two $\sum$-cospans as in Lemma 4.7, $\left(f_{1}, I_{1}, s_{1}\right) \equiv\left(f_{2}, I_{2}, s_{2}\right)$.

Next we define a composition between equivalence classes of $\sum$-cospans, for $\sum$ admitting a left calculus of lax fractions. We give the definition and we show that it is well-defined and that it is preserved by the order $\leq$ defined between equivalence classes of $\sum$-cospans.

Given two $\sum$-cospans $(f, I, s): A \rightarrow B$ and $(g, J, t): B \rightarrow C$, we define

$$
[(g, J, t)] \cdot[(f, I, s)]
$$

as being the equivalence class of any $\sum$-cospan $\left(g^{\prime} f, K, s^{\prime} t\right): A \rightarrow C$ obtained by forming a $\sum$-square as follows:

$$
\begin{aligned}
A \stackrel{f}{\longrightarrow} I \stackrel{s}{\longleftarrow} B \\
g^{\prime} \downarrow 3 \quad g \\
K \underset{s^{\prime}}{\longleftarrow} J \leftarrow_{t} C
\end{aligned}
$$

From now on a composition of two $\sum$-cospans $(f, I, s): A \rightarrow B$ and $(g, J, t): B \rightarrow C$ will be denoted by

$$
(g, J, t) \circ(f, I, s)
$$

and it refers to any $\sum$-cospan $\left(g^{\prime} f, K, s^{\prime} t\right): A \rightarrow C$ obtained as described above.

The above composition is well-defined, that is, if $I \stackrel{g^{\prime}}{\longrightarrow} K \stackrel{s^{\prime}}{\longleftarrow} J$ and $I \stackrel{\hat{s}}{\longrightarrow} M \stackrel{\hat{s}}{\leftarrow} J$ are two $\sum$-cospans covered by the $\sum$-span $I<s$

Indeed, in that case, by Lemma $4.7,\left(g^{\prime}, K, s^{\prime}\right) \leq(\hat{g}, M, \hat{s})$, thus we have a diagram of the form

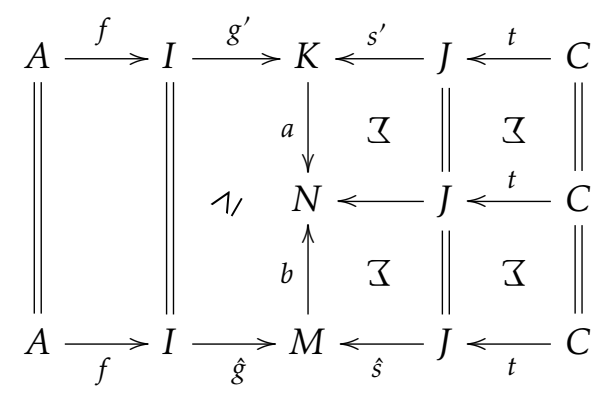

showing that $\left(g^{\prime} f, K, s^{\prime} t\right) \leq(\hat{g} f, M, \hat{s} t)$; and analogously, we have $(\hat{g} f, M, \hat{s} t) \leq\left(g^{\prime} f, K, s^{\prime} t\right)$.

Lemma 4.10. The relation $\leq$ is compactible with composition, i.e., if we have a diagram of $\sum$-cospans

$$
A \underset{\left(f_{1}, s_{1}\right)}{\stackrel{\left(f_{2}, s_{2}\right)}{\longrightarrow}} B \underset{\left(g_{1}, t_{1}\right)}{\longrightarrow} C
$$

with $\left(f_{1}, s_{1}\right) \leq\left(f_{2}, s_{2}\right)$ and $\left(g_{1}, t_{1}\right) \leq\left(g_{2}, t_{2}\right)$, then any composition of the two lower $\sum$-cospans is $\leq$-related to any composition of the two upper $\sum$-cospans.

Proof. It suffices to prove that the property holds for
(A) $(f, s)=\left(f_{1}, s_{1}\right)=\left(f_{2}, s_{2}\right)$, and
(B) $(g, t)=\left(g_{1}, t_{1}\right)=\left(g_{2}, t_{2}\right)$. 
(A) Let us have the inequality $\left(g_{1}, t_{1}\right) \leq\left(g_{2}, t_{2}\right)$ through the diagram

$$
\begin{aligned}
& B \stackrel{g_{1}}{\longrightarrow} J_{1} \stackrel{t_{1}}{\longleftarrow} C \\
& \begin{array}{c}
y_{1} \downarrow \\
1
\end{array} \|
\end{aligned}
$$

and, using Square, consider the compositions $\left(g_{i}, J_{i}, t_{i}\right) \circ(f, I, s), i=1,2$, given by

$$
\begin{aligned}
& A \stackrel{f}{\longrightarrow} I \stackrel{s}{<} B \\
& g_{i}^{\prime} \downarrow 3 \quad \downarrow g_{i} \\
& K_{i} \stackrel{s_{i}}{\longrightarrow} J_{i} \underset{t_{i}}{\longleftarrow} C
\end{aligned}
$$

Square also ensures the existence of the following first two $\sum$-squares, which in turn, combined with (16), give rise to the third diagram:

$$
\begin{aligned}
& J_{i} \stackrel{s_{i}}{\longrightarrow} K_{i} \quad, \quad i=1,2, \\
& J_{0} \stackrel{s_{1}^{\prime}}{\longrightarrow} L_{1} \\
& y_{i} \downarrow \sum \downarrow y_{i}^{\prime} \\
& J_{0} \underset{s_{i}^{\prime}}{\longrightarrow} L_{i} \\
& s_{2}^{\prime} \downarrow \quad \Sigma \quad \downarrow r^{r_{1}} \\
& L_{2} \underset{r_{2}}{\longrightarrow} M
\end{aligned}
$$

$$
\begin{gathered}
B \stackrel{s}{\longrightarrow} I \\
s_{2}^{\prime} y_{1} g_{1} \downarrow r_{1} y_{1}^{\prime} g_{1}^{\prime} \downarrow \downarrow r_{2} y_{2}^{\prime} g_{2}^{\prime} \\
L_{2} \underset{r_{2}}{\longrightarrow} M
\end{gathered} .
$$

In the last diagram the inner square is of $\Sigma$ type, because of Composition, and, furthermore, we have that $\left(r_{1} y_{1}^{\prime} g_{1}^{\prime}\right) s=r_{1} y_{1}^{\prime} s_{1} g_{1}=r_{1} s_{1}^{\prime} y_{1} g_{1}=r_{2} s_{2}^{\prime} y_{1} g_{1} \leq r_{2} s_{2}^{\prime} y_{2} g_{2}=r_{2} y_{2}^{\prime} s_{2} g_{2}=\left(r_{2} y_{2}^{\prime} g_{2}^{\prime}\right) s$. Consequently, by Coinsertion, there is $p: M \rightarrow P$ such that

$$
\begin{aligned}
& p r_{1} y_{1}^{\prime} g_{1}^{\prime} \leq p r_{2} y_{2}^{\prime} g_{2}^{\prime} \quad \text { and } \quad L_{2} \stackrel{r_{2}}{\longrightarrow} M \text {. } \\
& \| \sum \downarrow p \\
& L_{2} \underset{p r_{2}}{\longrightarrow} P
\end{aligned}
$$

Therefore, we have the following diagram, where $t=y_{i} t_{i}$,

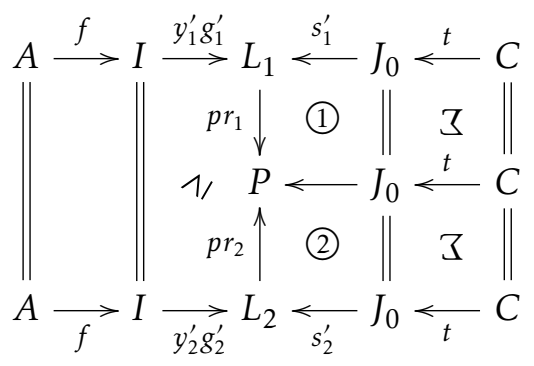


with both squares (1) and (2) of $\sum$ type. Indeed (1) and (2) are the outside squares of the following diagrams obtained by vertical and horizontal composition of $\sum$-squares:
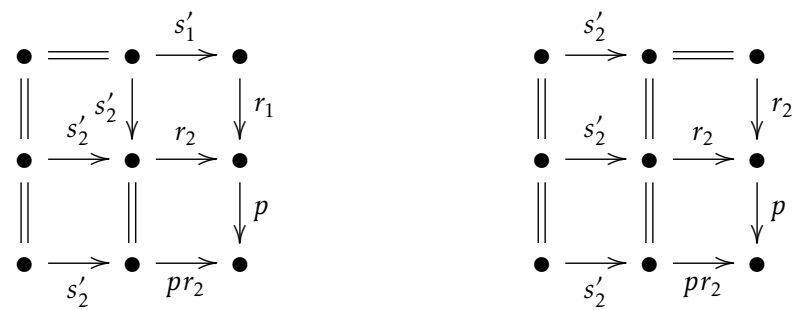

Using the first diagram of (17), and putting $t=y_{i} t_{i}$, we obtain the commutative diagram

$$
\begin{aligned}
& A \stackrel{f}{\longrightarrow} I \stackrel{g_{i}^{\prime}}{\longrightarrow} K_{i} \stackrel{s_{i}}{\longleftarrow} J_{i} \stackrel{t_{i}}{\longleftarrow} C .
\end{aligned}
$$

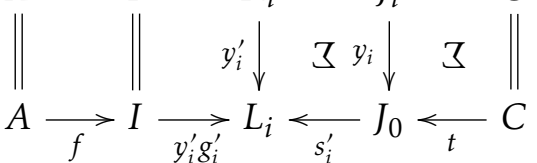

Now, the diagram obtained by composing vertically first the diagram (20) with $i=1$, next the diagram (19), and lastly the diagram (20) with $i=2$, shows that $\left(g_{1}^{\prime} f, s_{1} t_{1}\right) \leq\left(g_{2}^{\prime} f, s_{2} t_{2}\right)$, as desired.

(B) Let us have the inequality $\left(f_{1}, s_{1}\right) \leq\left(f_{2}, s_{2}\right)$ through the diagram

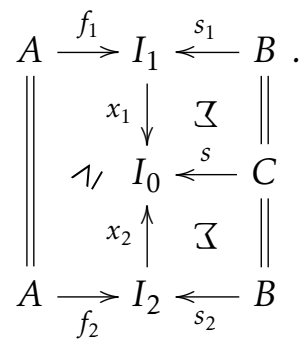

Then the following diagram, where $(\tilde{g}, \tilde{s})$ is a $\Sigma$-cospan obtained by Square applied to the $\Sigma$-span $(s, g)$,

$$
\begin{aligned}
& A \stackrel{f_{i}}{\longrightarrow} I_{i} \stackrel{s_{i}}{ } B \\
& x_{i} \downarrow 3 \\
& I_{0} \stackrel{s}{\longleftarrow} B \\
& \tilde{g} \downarrow 3 \downarrow g \\
& M \underset{\tilde{s}}{\longleftarrow} \longleftarrow_{t} C
\end{aligned}
$$

shows that, for $i=1,2,\left(\tilde{g} x_{i} f_{i}, \tilde{s} t\right)$ is a composition of $\left(f_{i}, s_{i}\right)$ with $(g, t)$. Thus, the diagram

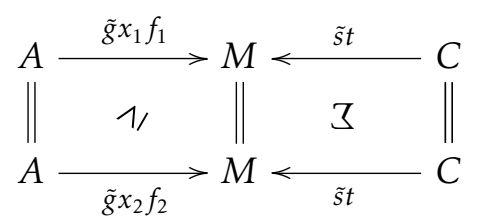

tells us that $(g, t) \circ\left(f_{1}, s\right) \leq(g, t) \circ\left(f_{2}, s\right)$.

Now we are ready to prove the announced theorem: 
Theorem 4.11. Let $\sum$ be a subcategory of $X \rightarrow$ admitting a left calculus of lax fractions. Then the category of lax fractions $P_{\Sigma}: X \rightarrow X\left[\Sigma_{*}\right]$ can be described as follows:

- the objects of $X\left[\Sigma_{*}\right]$ are those of $X$;

- the morphisms of $X\left[\Sigma_{*}\right]$ are $\equiv$-equivalence classes of $\Sigma$-cospans with the composition and order on morphisms as described in 4.9;

- $P_{\Sigma} A=A$ and $P_{\Sigma} f=[(f, i d)]$ for all objects $A$ and morphisms $f$ of $X$.

Proof. (A) $X\left[\Sigma_{*}\right]$, as described above, is actually a category.

The identity on an object $A$ is the equivalence class of $\left(\mathrm{id}_{A}, \mathrm{id}_{A}\right)$. Indeed, given $(f, I, s): A \rightarrow B$, using the fact that $\sum$ is a subcategory of $X \rightarrow$, Square and Identity, we obtain the diagrams

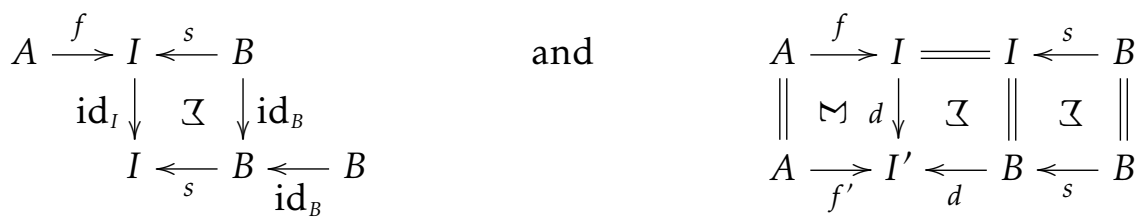

which show that $\left(\operatorname{id}_{B}, \operatorname{id}_{B}\right) \circ(f, s) \equiv(f, s)$ and $(f, s) \circ\left(\operatorname{id}_{A}, \operatorname{id}_{A}\right) \equiv\left(f^{\prime}, d s\right) \equiv(f, s)$.

Moreover, the associativity of the composition is illustrated by the following diagram, which shows that $\left(h^{\prime \prime} g^{\prime} f, s^{\prime \prime} t^{\prime} u\right)$ is simultaneously a composition of the form $((h, u) \circ(g, t)) \circ(f, s)$ and a composition of the form $(h, u) \circ((g, t) \circ(f, s))$ :

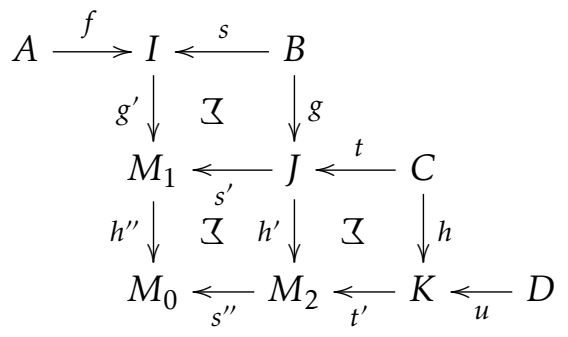

(B) $P_{\Sigma}$ is clearly a functor, since $P_{\Sigma}\left(\operatorname{id}_{A}\right)=\left(\operatorname{id}_{A}, \operatorname{id}_{A}\right)$, and, given $f: A \rightarrow B$ and $g: B \rightarrow C$ in $X$, we have that $P_{\Sigma}(g) \cdot P_{\Sigma}(f) \equiv\left(g, \operatorname{id}_{C}\right) \circ\left(f, \operatorname{id}_{B}\right) \equiv\left(g f, \operatorname{id}_{C}\right) \equiv P_{\Sigma}(g f)$; to see that indeed $\left(g, \operatorname{id}_{C}\right) \circ\left(f, \operatorname{id}_{B}\right) \equiv$ $\left(g f, \operatorname{id}_{C}\right)$, let $\left(g^{\prime} f, d\right)$ be a composition of $\left(g\right.$,id) with $(f$, id $)$, i.e., $\left(g, g^{\prime}\right):$ id $\rightarrow d$ is a morphism of $\Sigma$, obtained by Square; then, using Identity, we have the diagram

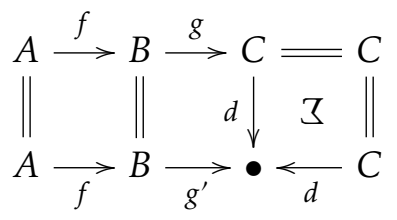

which shows that $\left[\left(g f, \operatorname{id}_{C}\right)\right]=\left[\left(g, \operatorname{id}_{C}\right)\right] \cdot\left[\left(f, \operatorname{id}_{B}\right)\right]$.

Furthermore, $P_{\Sigma}$ is order-enriched: given $f, g: A \rightarrow B$ with $f \leq g$, then $P_{\Sigma} f \leq P_{\Sigma} g$.

(C) To verify that $P_{\Sigma}$ satisfies condition (i) of Definition 3.1, let $s: A \rightarrow B$ be an object of $\Sigma$. We show that $P_{\Sigma} s=\left[\left(s, \mathrm{id}_{B}\right)\right]$ is a left adjoint section, by showing that $\left[\left(\operatorname{id}_{B}, s\right)\right] \cdot\left[\left(s, \operatorname{id}_{B}\right)\right]=\left[\left(\operatorname{id}_{A}, \operatorname{id}_{A}\right)\right]$ and $\left(s, \mathrm{id}_{B}\right) \circ\left(\operatorname{id}_{B}, s\right) \leq\left(\operatorname{id}_{B}, \mathrm{id}_{B}\right)$; thus, in particular, we have that $([(s, \mathrm{id})])_{*}=[(\mathrm{id}, s)]$. The $\sum$-cospan $(s, s)$ is clearly a composition of the form $\left(\operatorname{id}_{B}, s\right) \circ\left(s, \mathrm{id}_{B}\right)$, and the fact that $(s, s) \equiv\left(\operatorname{id}_{A}, \mathrm{id}_{A}\right)$ follows 
from the diagram

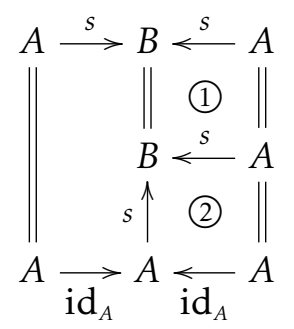

where (1) is a $\sum$-square because it is the identity morphism on the object $s$ of $\Sigma$, and (2) is a $\sum$ square because of Identity. In order to conclude that $\left(s, \operatorname{id}_{B}\right) \circ\left(\operatorname{id}_{B}, s\right) \leq\left(\operatorname{id}_{B}, \operatorname{id}_{B}\right)$, let $\left(s_{1}, s_{2}\right)$ be a composition of $\left(s, \mathrm{id}_{B}\right)$ with $\left(\mathrm{id}_{B}, s\right)$, as illustrated by the following diagram:

$$
\begin{aligned}
B \stackrel{\mathrm{id}_{B}}{\longrightarrow} B \stackrel{s}{ } & A \\
s_{1} \downarrow & 3 \downarrow \checkmark s \\
& \quad \underset{s_{2}}{\leftarrow} B \underset{\mathrm{id}_{B}}{\longleftarrow} B
\end{aligned}
$$

Since $s_{1} s=s_{2} s$, by Coinsertion we know that there is a morphism $d: C \rightarrow D$ such that $d s_{1} \leq d s_{2}$ and the $\sum$-span $\left(s_{2}, \mathrm{id}_{B}\right)$ covers the $\sum$-cospan $\left(d, d s_{2}\right)$. We obtain then the diagram

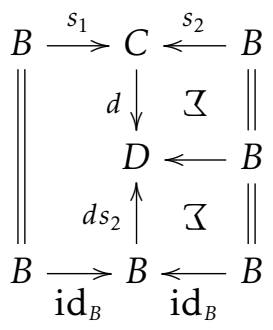

with $d s_{1} \leq d s_{2}$. That is, $\left(s_{1}, s_{2}\right) \leq\left(\operatorname{id}_{B}, \operatorname{id}_{B}\right)$, where $\left(s_{1}, s_{2}\right)$ is a representative of $\left[\left(s, \mathrm{id}_{B}\right) \circ\left(\mathrm{id}_{B}, s\right)\right]$.

Now, the satisfaction of (ii) of Definition 3.1 is easily seen since, given a morphism $(u, v): r \rightarrow s$ in $\Sigma$, it is clear that $(u, \mathrm{id}) \circ(\mathrm{id}, r) \equiv(v, s) \equiv(\mathrm{id}, s) \circ(v, \mathrm{id})$.

(D) $P_{\Sigma}$ is universal. Let $F: \mathcal{X} \rightarrow \mathcal{C}$ be a functor such that $F s$ is a left adjoint section for every $s \in \Sigma$, and, moreover, for every morphism $(f, g): r \rightarrow s$ in $\Sigma$, the equality $(F s)_{*} g=f(F r)_{*}$ holds. We define $H: X\left[\Sigma_{*}\right] \rightarrow \mathcal{C}$ by

$$
H X=F X \quad \text { and } \quad H[(f, I, s)]=(F s)_{*} F f .
$$

First we show that, assuming that $H$ is a functor, it is the unique one such that $H P_{\Sigma}=F$. Indeed we have $H\left(P_{\Sigma} X\right)=H X=F X$; and $H\left(P_{\Sigma} f\right)=H(f$,id $)=(F(\text { id }))_{*} F f=F f$. Furthermore, if $\bar{H}: X\left[\Sigma_{*}\right] \rightarrow$ $\mathcal{C}$ is another functor such that $\bar{H} P_{\Sigma}=F$, taking into account that we are dealing with order-enriched functors, we have that:

$$
\begin{aligned}
\bar{H} X & =\bar{H}\left(P_{\Sigma} X\right)=F X ; \text { and } \\
\bar{H}[(f, I, s)] & =\bar{H}\left[\left(\operatorname{id}_{I}, I, s\right)\right] \cdot \bar{H}\left[\left(f, I, \mathrm{id}_{I}\right)\right] \\
& =\left(\bar{H}\left[\left(s, I, \mathrm{id}_{I}\right)\right]\right)_{*} \cdot \bar{H}\left[\left(f, I, \mathrm{id}_{I}\right)\right] \\
& =\left(\bar{H} P_{\Sigma} s\right)_{*} \cdot\left(\bar{H} P_{\Sigma} f\right) \\
& =(F s)_{*} F f \\
& =H[(f, I, s)] .
\end{aligned}
$$

It remains to show that $H: X\left[\sum_{*}\right] \rightarrow \mathcal{C}$ is indeed a functor. 
$H$ is well-defined on equivalence classes and is order-enriched. In order to conclude these both assertions, taking into account that $\equiv$ is defined by means of $\leq$, it suffices to prove that, for a pair of $\sum$-cospans $(f, I, s),(g, J, t): A \rightarrow B$ with $(f, I, s) \leq(g, J, t)$, we have that $(F s)_{*} F f \leq(F t)_{*} F g$. Indeed, if $(f, I, s) \leq(g, J, t)$, then we have a diagrama as follows:

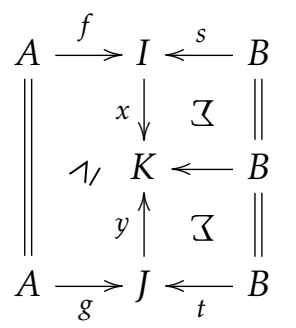

The fact that the two squares on the right-hand side are of $\sum$ type implies that $(F(x s))_{*} F x=(F s)_{*}$ and $(F t)_{*}=(F(y t))_{*} F y$, by assumption on $F$. Hence,

$$
(F s)_{*} F f=(F(x s))_{*} F x F f \leq(F(x s))_{*} F y F g=(F(y t))_{*} F y F g=(F t)_{*} F g .
$$

$H$ is functorial. Indeed, $H$ preserves identities since $H\left[\left(\mathrm{id}_{A}, \mathrm{id}_{A}\right)\right]=\left(F \mathrm{id}_{A}\right)_{*}\left(F \mathrm{Fid}_{A}\right)=\mathrm{id}_{F A}$. In order to show that $H$ preserves composition, given $\sum$-cospans $(f, s): A \rightarrow B$ and $(g, t): B \rightarrow C$, let $(\tilde{g} f, \tilde{s} t)$ be a composition of them, that is, $\stackrel{s}{\longrightarrow} \bullet$. Then we have that

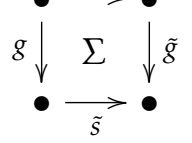

$H([(g, t)] \cdot[(f, s)])=H([(\tilde{g} f, \tilde{s} t)])=(F(\tilde{s} t))_{*} F(\tilde{g} f)=(F t)_{*}(F \tilde{s})_{*} F \tilde{g} F f$. But, by hypothesis, $(F \tilde{s})_{*} F \tilde{g}=$ $F g(F s)_{*}$. Consequently, we obtain $H([(g, t)] \cdot[(f, s)])=(F t)_{*} F g(F s)_{*} F f=H([(g, t)]) \cdot H([(f, s)])$.

\section{The COCOMPLeteness of $\mathcal{A}$ Llnj}

We recall from [17] that an order-enriched category $X$ has weighted colimits if and only if it has conical coproducts and coinserters. We also recall that $X$ has conical coproducts whenever it has coproducts and the corresponding injections are collectively order-epic, that is, for every coproduct $v_{i}: X_{i} \rightarrow \bigsqcup_{i \in I} X_{i}$ and every pair of morphisms $f, g: \bigsqcup_{i \in I} X_{i} \rightarrow Y$ with $f v_{i} \leq g v_{i}, i \in I$, we have $f \leq g$. The coinserter of a pair of morphisms $f, g: X \rightarrow Y$ is an order-epic morphism $c: Y \rightarrow C$ such that $c f \leq c g$ and every morphism $d: Y \rightarrow D$ with $d f \leq d g$ factorizes uniquely through $c$; briefly, $c=\operatorname{coins}(f, g)$.

If $X$ has weighted colimits, then the arrow category $X \rightarrow$ also has weighted colimits, and they are constructed coordinatewise. We are going to see that $\mathcal{A} \underline{\text { LInj }}$ is closed under weighted colimits in $x \rightarrow$.

Theorem 5.1. Let $X$ have weighted colimits. Then, for every subcategory $\mathcal{A}$ of $X$, the category $\mathcal{A}$ ㄴǌ is closed under weighted colimits in $x \rightarrow$.

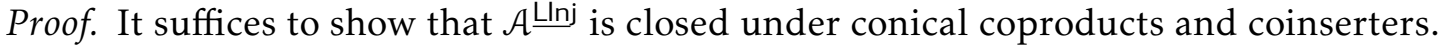

Concerning conical coproducts, let $h_{i}: X_{i} \rightarrow Y_{i}$ belong to $\mathcal{A}$ ㄴǌ , and form the conical coproduct in $x \rightarrow$ :

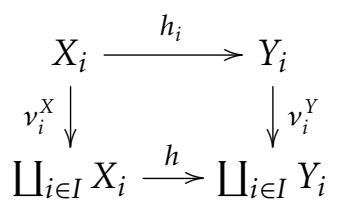


First we show that $h \in \mathcal{A} \underline{\text { LInj }}$ and $\left(v_{i}^{X}, v_{i}^{Y}\right)$ are morphisms of $\mathcal{A}$ 늘. Given $g: \bigsqcup_{i \in I} X_{i} \rightarrow A$, with $A \in \mathcal{A}$, put:

$$
g / h: \coprod_{i \in I} Y_{i} \rightarrow A \text { is the unique morphism such that }(g / h) v_{i}^{Y}=\left(g v_{i}^{X}\right) / h_{i}, i \in I .
$$

We show that $g / h$ deserves its designation. Indeed,

$$
(g / h) h v_{i}^{X}=(g / h) v_{i}^{Y} h_{i}=\left(\left(g v_{i}^{X}\right) / h_{i}\right) h_{i}=g v_{i}^{X}, i \in I,
$$

hence $(g / h) h=g$. And, for $s: \bigsqcup_{i \in I} Y_{i} \rightarrow A$ with $g \leq s h$, we have $g v_{i}^{X} \leq s h v_{i}^{X}=s v_{i}^{Y} h_{i}$, thus $\left(g v_{i}^{X}\right) / h_{i} \leq$ $s v_{i}^{Y}$, that is, $(g / h) v_{i}^{Y} \leq s v_{i}^{Y}$. Since this holds for all $i, g / h \leq s$. Moreover, since $g / h$ is defined by (22), it is clear that all $\left(v_{i}^{X}, v_{i}^{Y}\right)$ are morphisms of $\mathcal{A}$ 느․

Let now have morphisms $\left(r_{i}, s_{i}\right): h_{i} \rightarrow t$ in $\mathcal{A}^{\text {LInj }}, i \in I$. Then, in $X^{\rightarrow}$, we have a unique morphism $(r, s): h \rightarrow t$ such that $(r, s) \cdot\left(v_{i}^{X}, v_{i}^{Y}\right)=\left(r_{i}, s_{i}\right), i \in I$ :

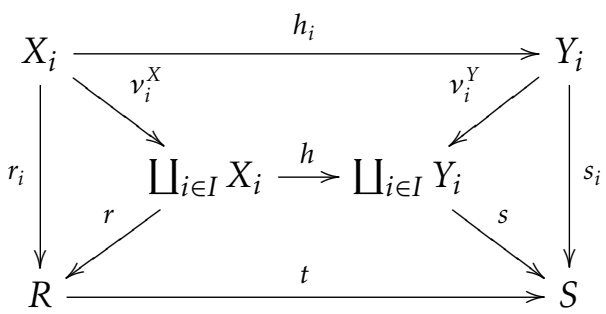

We show that $(r, s)$ is a morphism of $\mathcal{A} \underline{\text { Llnj }}$. Consider $a: R \rightarrow A$ with $A \in \mathcal{A}$. Then, using the fact that $\left(v_{i}^{X}, v_{i}^{Y}\right)$ and $\left(r_{i}, s_{i}\right)$ are both morphisms of $\mathcal{A}^{\text {LInj }}$ and formula (22), we have:

$$
(a / t) s v_{i}^{Y}=(a / t) s_{i}=\left(a r_{i}\right) / h_{i}=\left(a r v_{i}^{X}\right) / h_{i}=((a r) / h) v_{i}^{Y} .
$$

Consequently, $(a / t) s=(a r) / h$.

Concerning coinserters, let $\left(u_{1}, v_{1}\right),\left(u_{2}, v_{2}\right): f \rightarrow g$ be two morphisms in $\mathcal{A}$ Llnj and let $(c, d)$ be the coinserter of $\left(\left(u_{1}, v_{1}\right),\left(u_{2}, v_{2}\right)\right)$ in $X \rightarrow$ :

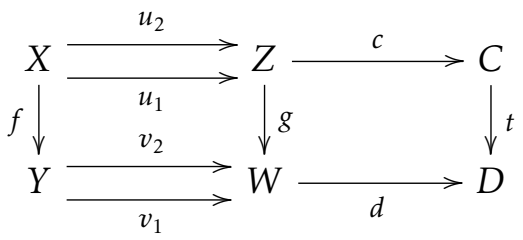

In particular, $c=\operatorname{coins}\left(u_{1}, u_{2}\right), d=\operatorname{coins}\left(v_{1}, v_{2}\right)$, and $t$ is the unique morphism for which $t c=d g$. We want to show that the morphism $(c, d)$ is also the coinserter of $\left(u_{1}, v_{1}\right)$ and $\left(u_{2}, v_{2}\right)$ in $\mathcal{A}$ ㄴǌ.

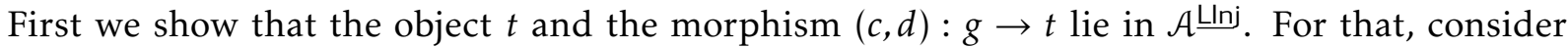
$k: C \rightarrow A$ with $A$ in $\mathcal{A}$. Taking into account that $\left(u_{i}, v_{i}\right), i=1,2$, are morphisms in $\mathcal{A}$ Llnj , and that $c u_{1} \leq c u_{2}$, we have that

$$
((k c) / g) v_{1}=\left(k c u_{1}\right) / f \leq\left(k c u_{2}\right) / f \leq((k c) / g) v_{2},
$$

and, consequently, since $d=\operatorname{coins}\left(v_{1}, v_{2}\right)$, there is a unique morphism $w: D \rightarrow A$ with

$$
w d=(k c) / g .
$$

We show that $w=k / t$. Indeed, $w t c=w d g=((k c) / g) g=k c$, thus $w t=k$, since $c$ is order-epic, in particular, an epimorphism. Moreover, if $w^{\prime}: D \rightarrow A$ is such that $k \leq w^{\prime} t$, then $k c \leq w^{\prime} t c=w^{\prime} d g$, 
then $(k c) / g \leq w^{\prime} d$, and we have that $w d=(k c) / g \leq w^{\prime} d$. Now, since $d$ is order-epic, it follows that $w \leq w^{\prime}$

The conclusion that $(c, d): g \rightarrow t$ is a morphism in $\mathcal{A} \underline{\underline{L} n \mathrm{n}}$ is immediate from the definition of $w$ in (25).

Let us now have $t^{\prime}: C^{\prime} \rightarrow D^{\prime}$ and a morphism $\left(c^{\prime}, d^{\prime}\right): g \rightarrow t^{\prime}$ in $\mathcal{A}$ Llnj with $\left(c^{\prime}, d^{\prime}\right) \cdot\left(u_{1}, v_{1}\right) \leq$ $\left(c^{\prime}, d^{\prime}\right) \cdot\left(u_{2}, v_{2}\right)$.

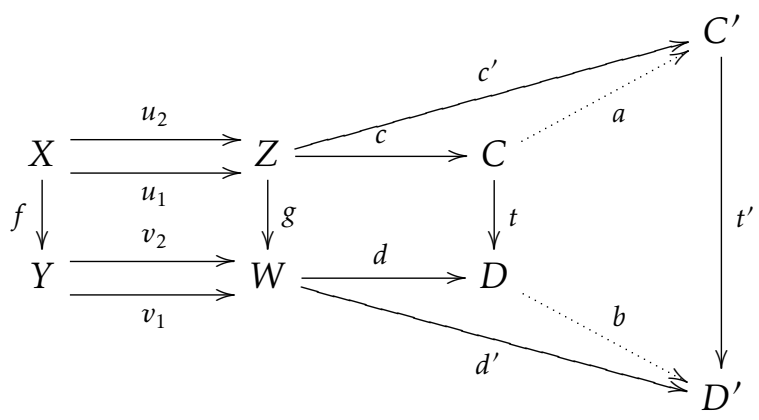

Since $(c, d)=$ coins $\left(\left(u_{1}, v_{1}\right),\left(u_{2}, v_{2}\right)\right)$ in $X \rightarrow$, there is a unique morphism $(a, b): t \rightarrow t^{\prime}$ such that $(a c, b d)=\left(c^{\prime}, d^{\prime}\right)$. We want to show that $(a, b)$ lies in $\mathcal{A}$ Llnj. Let then $l: C^{\prime} \rightarrow A$ have codomain in $\mathcal{A}$. From above, we know that $(l a) / t$ is the unique morphism such that $((l a) / t) d=(l a c) / g$. But, by hypothesis, $\left(l / t^{\prime}\right) b d=(l a c) / g$, thus $\left(l / t^{\prime}\right) b d=((l a) / t) d$ and, consequently, $\left(l / t^{\prime}\right) b=(l a) / t$, as desired.

Remark 5.2. Moreover, under the conditions of the above theorem, $\mathcal{A}$ Llnj is a coinserter-ideal. That is, given a parallel pair of morphisms $\left(u_{1}, v_{1}\right),\left(u_{2}, v_{2}\right): f \rightarrow g$ in $X \rightarrow$, if $\left(u_{1}, v_{1}\right)$ belongs to $\mathcal{A}$ Llnj then also the coinserter of $\left(\left(u_{1}, v_{1}\right),\left(u_{2}, v_{2}\right)\right)$ lies in $\mathcal{A} \underline{\text { LInj }}$. Indeed, in the above proof of the closedness of $\mathcal{A} \underline{\text { LInj }}$ under coinserters we only used the fact that $\left(u_{1}, v_{1}\right)$ belongs to $\mathcal{A} \underline{\underline{\text { Lnj }}}$.

Next we show that the existence of finite weighted colimits in $X$ allows $\mathcal{A} \stackrel{\text { Llnj }}{\text { to admit a left }}$ calculus of lax fractions.

Proposition 5.3. Let $X$ have finite weighted colimits and let $\mathcal{A}$ be a subcategory of $X$. Then $\Sigma=\mathcal{A} \underline{\text { Linj }}$ admits a left calculus of lax fractions.

Proof. Identity is obvious, since we always have that, supposing that $g / s$ is defined, $(g \cdot \mathrm{id}) / \mathrm{id}=g=$ $(g / s) s$.

Concerning Composition, given two $\Sigma$-squares as the two first ones in Definition 4.2.2, let $a: \bullet \rightarrow$ $A$, with $A$ in $\mathcal{A}$, be composable with $f$. It is easy to see that, given a composition $\bullet \stackrel{s}{\longrightarrow} \bullet \stackrel{s^{\prime}}{\longrightarrow} \bullet$

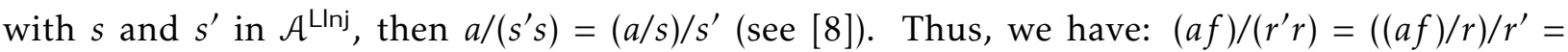
$((a / s) g) / r^{\prime}=\left((a / s) / s^{\prime}\right) h=\left(a /\left(s^{\prime} s\right)\right) h$. 
To obtain Square, we show that every pushout

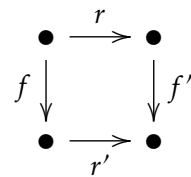

follows from the closedness of $\mathcal{A}^{\text {LInj }}$ under pushouts proven in [8], and can be derived from Theorem 5.1: the diagram $\quad \mathrm{id} \stackrel{(\mathrm{id}, r)}{\longrightarrow} r \quad$ is a pushout in $X \rightarrow$, and $(\mathrm{id}, r)$ and $(f, f)$ are easily seen<smiles>[Al][Te][Te][Te][Te]</smiles>

to be morphisms in $\mathcal{A}$ Llnj; thus, by the above theorem the same holds to $\left(f, f^{\prime}\right): r \rightarrow r^{\prime}$.

To show Coinsertion, given a diagram $U \stackrel{r}{\longrightarrow} V$ with the inner square of $\sum$ type and $g r \leq h r$,

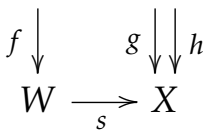

let $t: X \rightarrow T$ be the coinserter of $(g, h)$. Thus $t g \leq t h$. We show that the morphism $t s$ lies in $\Sigma$ and (id, $t): s \rightarrow t s$ is a morphism of $\Sigma$. Indeed, given $a: W \rightarrow A$ with $A \in \mathcal{A}$, af $=(a / s) s f=$ $(a / s) g r \leq(a / s) h r$, thus $(a f) / r \leq(a / s) h$. But, by hypothesis, $(a f) / r=(a / s) g$. Thus, $(a / s) g \leq(a / s) h$ and, consequently, there is a unique morphism $u: T \rightarrow A$ such that $u t=a / s$. It is easy to see that $u=a /(t s)$. For, if, for $v: T \rightarrow A$, we have $a \leq v(t s)$, then $a / s \leq v t$, that is, $u t \leq v t$, and, since $t$ is an order-epimorphism, $u \leq v$. Moreover, we have $(a \cdot \mathrm{id}) / s=a / s=u t=(a /(t s)) t$, that is, $(\mathrm{id}, t): s \rightarrow t s$ is a morphism of $\sum$.

In the ordinary case, we know that if $\Sigma$ is a class of morphisms of a finitely cocomplete category $X$ admitting a left calculus of fractions then the category of fractions $X\left[\Sigma^{-1}\right]$ has finite colimits ([13]).

In the following we see that if $X$ has finite conical coproducts then, for $\Sigma$ a subcategory of $X \rightarrow$ admitting a left calculus of lax fractions and satisfying an extra condition, $\mathcal{X}\left[\Sigma_{*}\right]$ has finite conical coproducts too. Moreover, if $\mathcal{X}$ has weighted colimits then any (quasi)category $\mathcal{X}\left[\Sigma_{*}\right]$ with $\Sigma=\mathcal{A}$ Llnj has (small) conical coproducts.

Definition 5.4. For $X$ an order-enriched category, a subcategory $\sum$ of $X \rightarrow$ is said to satisfy the Coequalization condition if given two $\Sigma$-squares $U \stackrel{r}{\longrightarrow} V, i=1,2$, there exists some morphism

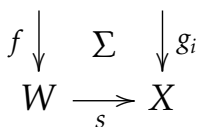

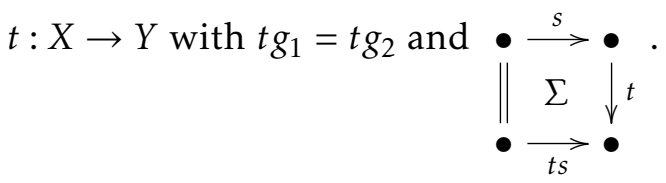

Remark 5.5. 1. Let $X$ have weighted colimits. An argument similar to the one used for Coinsertion in the proof of Proposition 5.3 shows that $\mathcal{A} \stackrel{\underline{L} \ln }{ }$ also satisfies Coequalization, for every subcategory $\mathcal{A}$ of $X$.

2. Let $\Sigma$ be a subcategory of $X \rightarrow$ satisfying the four conditions of a left calculus of lax fractions together with Coequalization. Then, by using arguments analogous to the ones of the proof of Lemma 4.7, we conclude that, given two $\Sigma$-cospans $(f, s)$ and $(g, t)$ from $A$ to $B$, we have that 
$(f, s) \equiv(g, t)$ if and only if there is a commutative diagram of the following form:

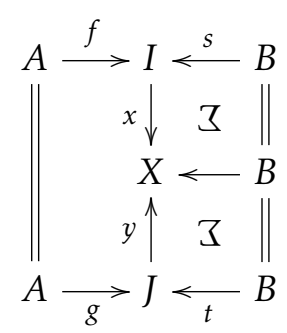

Proposition 5.6. 1. If $X$ has weighted colimits and $\Sigma=\mathcal{A}$ ㄴǌ for some subcategory $\mathcal{A}$ of $X$, then the (quasi)category $X\left[\Sigma_{*}\right]$ has, and $P_{\Sigma}$ preserves, (small) conical coproducts.

2. If $X$ has finite conical coproducts and $\Sigma$ is a subcategory of $X \rightarrow$ satisfying the four conditions of a left calculus of lax fractions together with Coequalization, then $X\left[\Sigma_{*}\right]$ has, and $P_{\Sigma}$ preserves, finite conical coproducts.

Proof. 1. Given $X_{i} \in \mathcal{X}\left[\Sigma_{*}\right], i \in I$, let $v_{i}: X_{i} \rightarrow \bigsqcup_{i \in I} X_{i}$ be a conical coproduct in $\mathcal{X}$. We show that $\left[\left(v_{i}, \mathrm{id}\right)\right]: X_{i} \rightarrow \bigsqcup_{i \in I} X_{i}$ constitutes a conical coproduct in $X\left[\Sigma_{*}\right]$. First, we see that the morphisms $\left[\left(v_{i}\right.\right.$, id $\left.)\right]$ are collectively order-epic. For that, let us have two $\Sigma$-cospans

$$
\coprod_{i \in I} X_{i} \stackrel{(g, J, t)}{\longrightarrow} Y
$$

with $(f, s) \circ\left(v_{i}, \mathrm{id}\right) \leq(g, t) \circ\left(v_{i}\right.$, id $)$. It is easy to see that $\left(f v_{i}, s\right) \equiv(f, s) \circ\left(v_{i}\right.$, id $)$, since, for $(\mathrm{id}, d): f \rightarrow$ $f^{\prime}$ a morphism of $\sum$ given by Square, we have $\left(f v_{i}, s\right) \equiv\left(f^{\prime} v_{i}, d s\right)$. Analogously for $\left(g v_{i}, s\right)$. Thus $\left(f v_{i}, s\right) \leq\left(g v_{i}, t\right)$. We show that then $(f, s) \leq(g, t)$. By hypothesis, there are diagrams of the form

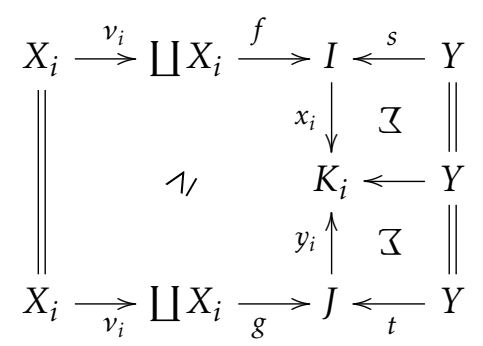

where all morphisms $x_{i} s\left(=y_{i} t\right)$ are objects of $\Sigma$. Since, by Identity, the morphisms $\left(\operatorname{id}_{Y}, x_{i} s\right): \operatorname{id}_{Y} \rightarrow$ $\left(x_{i} s\right)$ of $X^{\rightarrow}$ lie in $\Sigma=\mathcal{A} \underline{\text { LInj }}$, it follows from Theorem 5.1 that their wide pushout

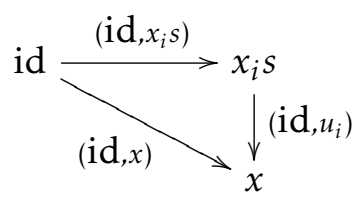

also lies in $\Sigma$. In particular, we have $\Sigma$-squares $\stackrel{x_{i} s}{\longrightarrow} \bullet$; and then, by vertical composition $\underset{x}{\stackrel{\sum}{\longrightarrow} K}$

of $\sum$-squares, we also have $Y \stackrel{s}{\longrightarrow} I \quad$ with $u_{i} x_{i} s=u_{j} x_{j} s$ for all $i, j \in I$. Let $c: X \rightarrow C$ be the

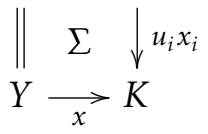


coequalizer of all morphisms $u_{i} x_{i}$. Then (id, $\left.c\right): x \rightarrow c x$ is the coequalizer of all (id, $\left.u_{i} x_{i}\right): s \rightarrow x$ in $\sum$, and, in particular, we obtain the $\sum$-square $Y \stackrel{x}{\longrightarrow} K$. Now we have that $c u_{i} x_{i} f v_{i} \leq c u_{i} y_{i} g v_{i}$,

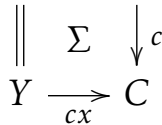

with $c u_{i} x_{i}=c u_{j} x_{j}, i, j \in I$. Since $\left(\mathrm{id}, u_{i}\right): y_{i} t=x_{i} s \rightarrow x$ is a morphism of $\sum$ (see (27)), using vertical composition, we also obtain the $\Sigma$-square $Y \stackrel{t}{\longrightarrow} J \quad$ with $c u_{i} y_{i} t=c u_{j} y_{j} t, i, j \in I$. Consequently,

$$
\begin{aligned}
& \| \sum \downarrow c u_{i} y_{i} \\
& Y \underset{c x}{\longrightarrow} C
\end{aligned}
$$

for the coequalizer $d: C \rightarrow D$ of all morphisms $c u_{i} y_{i}$ we have that all morphisms $d c u_{i} y_{i}$ are equal and $Y \stackrel{c x}{\longrightarrow} C$. Putting $a=d c u_{i} x_{i}$ and $b=d c u_{i} y_{i}$, it follows that $a f v_{i} \leq b g v_{i}$ for all $i$; then<smiles>[Y][Te]=C</smiles>

$a f \leq b g$. Now we have the diagram

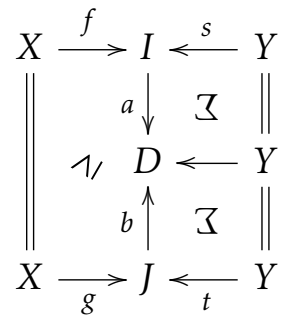

which shows that $(f, I, s) \leq(g, J, t)$, as desired.

Let now $\left(f_{i}, I_{i}, s_{i}\right): X_{i} \rightarrow Y$ be a family of $\sum$-cospans indexed by $I$. Let

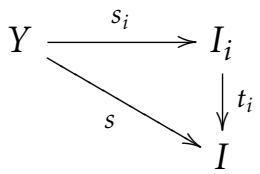

be the wide pushout of the morphisms $s_{i}: Y \rightarrow I_{i}$ in $X$. Then, by Theorem 5.1, arguing as for (27), we obtain the $\sum$-square $Y \stackrel{s_{i}}{\rightarrow} I_{i}$. By the universality of the coproduct in $X$, there is $\|_{Y \underset{s}{\longrightarrow} I}{ }^{\longrightarrow} t_{i}$

a unique morphism $w: \bigsqcup X_{i} \rightarrow I$ in $X$ with $w v_{i}=t_{i} f_{i}$, for all $i$. Then, composing $\sum$-cospans, we have: $(w, s) \circ\left(v_{i}, \mathrm{id}\right) \equiv\left(w v_{i}, s\right)=\left(t_{i} f_{i}, s\right) \equiv\left(f_{i}, s_{i}\right)$. Hence $[(w, s)]$ is a morphism of $X\left[\sum_{*}\right]$ with $[(w, s)] \cdot\left[\left(v_{i}, \mathrm{id}\right)\right]=\left[\left(f_{i}, s_{i}\right)\right]$. The uniqueness of $[(w, s)]$ follows from the fact already proved that the morphisms $\left[\left(v_{i}\right.\right.$, id $\left.)\right]$ are collectively order-epic.

By the above description of the coproducts in $X\left[\Sigma_{*}\right]$ it is clear that $P_{\Sigma}$ preserves coproducts.

2. The fact that $X\left[\Sigma_{*}\right]$ has binary coproducts is proved in a completely analogous way to 1 . Just in the situations where we needed to construct a wide pushout, we use now Square, and in the places where we needed coequalizers, we use Coequalization. It is easy to see that the initial object of $X$ is also the initial object of $X\left[\Sigma_{*}\right]$.

Remark 5.7. We leave as an open question the existence of coinserters in $\mathcal{X}\left[\Sigma_{*}\right]$ for $\Sigma=\mathcal{A}$ 늠 , when $X$ has weighted colimits. 


\section{REFERENCES}

[1] J. Adámek, L. Sousa, J. Velebil, Kan injectivity in order-enriched categories, Math. Structures Comput. Sci. 25 (2015), no. 1, 6-45.

[2] J. Adámek, L. Sousa, J. Velebil, KZ-monadic categories and their logic, manuscript.

[3] J. Adámek, M. Hébert, L. Sousa, A logic of orthogonality, Archivum Mathematicum 42, no.4, (2006), 309-334.

[4] J. Adámek, M. Sobral, L. Sousa, A logic of implications in algebra and coalgebra, Algebra Univers. 61 (2009) 313337.

[5] F. Borceux, Handbook of Categorical Algebra, Vol. 1, 2, Cambridge Univ. Press, 1994.

[6] B. Banaschewski and G. Bruns, Categorical characterisation of the MacNeille completion, Arch. Math. (Basel) 18 (1967), 369-377.

[7] J. Bénabou, Some geometric aspects of the calculus of fractions, Appl. Categor. Struct. 4 (1996), 139-165.

[8] M. Carvalho and L. Sousa, Order-preserving reflectors and injectivity, Topology Appl. 158 (2011), no. 17, 24082422.

[9] M. Carvalho and L. Sousa, On Kan-injectivity of locales and spaces, accepted for publication in Appl. Categor. Struct. (2015), 10.1007/s10485-015-9413-z.

[10] R. J. M. Dawson, R. Paré, D. A. Pronk, Adjoining adjoints, Adv. in Math. 178 (2003), pp. 99-140.

[11] R. J. M. Dawson, R. Paré, D. A. Pronk, Undecidability of the free adjoint construction, Appl. Categor. Struct. 11 (2003), pp. 403-419.

[12] M. H. Escardó, Properly injective spaces and function spaces, Topology Appl. 89(1-2) (1998), 75 -120.

[13] P. Gabriel and M. Zisman, Calculus of Fractions and Homotopy Theory, Springer Verlag 1967.

[14] E. Giuli, K.A. Hardie and J.J.C. Vermeulen, The Kleisli category is a category of fractions, Quaest. Math. 25 (2002), no. 3, 397-403.

[15] P. T. Johnstone, Stone Spaces, Cambridge University Press, Cambridge, 1982.

[16] P. T. Johnstone, Sketches of an Elephant: A Topos Theory Compendium, vol. 2. Number 44 in Oxford Logic Guides, The Clarendon Press, Oxford University Press, Oxford, 2002.

[17] G. M. Kelly, Basic concepts of enriched category theory, London Math. Soc. Lecture Notes Series 64, Cambridge Univ. Press, 1982, Repr. Theory Appl. Categ. 10 (2005), 1-136.

[18] A. Kock, Monads for which structures are adjoint to units (version 3), J. Pure Appl. Algebra 104 (1995), 41-59.

[19] S. MacLane, Categories for the Working Mathematician, 2nd ed., Springer-Verlag, Berlin-Heidelberg-New York 1998.

[20] R. Quackbenbush, Completeness theorems for universal algebra and implicational logics of algebras via congruences, Proc. Amer. Math. Society 103 (1988) 1015-1021.

[21] W. Tholen, Factorizations, localizations, and the orthogonal subcategory problem, Math Nachr. 114 (1983), 63-85.

Centre for Mathematics of the University of Coimbra \& Polytechnic Institute of Viseu, Portugal

E-mail address, corresponding author: sousa@estv.ipv.pt 